\title{
EDGE DIRECTION PRESERVING IMAGE ZOOMING: A MATHEMATICAL AND NUMERICAL ANALYSIS*
}

\author{
F. MALGOUYRES ${ }^{\dagger}$ AND F. GUICHARD $\ddagger$
}

\begin{abstract}
We focus in this paper on some reconstruction/restoration methods whose aim is to improve the resolution of digital images. The main point here is to study the ability of such methods to preserve one-dimensional (1D) structures. Indeed, such structures are important since they are often carried by the image "edges." First we focus on linear methods, give a general framework to design them, and show that the preservation of $1 \mathrm{D}$ structures pleads in favor of the cancellation of the periodization of the image spectrum. More precisely, we show that preserving 1D structures implies the linear methods to be written as a convolution of the "sinc interpolation." As a consequence, we cannot cope linearly with Gibbs effects, sharpness of the results, and the preservation of the 1D structure. Second, we study variational nonlinear methods and, in particular, the one based on total variation. We show that this latter permits us to avoid these shortcomings. We also prove the existence and consistency of an approximate solution to this variational problem. At last, this theoretical study is highlighted by experiments, both on synthetic and natural images, which show the effects of the described methods on images as well as on their spectrum.
\end{abstract}

Key words. image reconstruction, image restoration, image interpolation, Fourier analysis, variational methods, total variation, numerical approximation

AMS subject classifications. 68U10, 65D15, 65C20, 49R99, 42B05

\section{PII. S0036142999362286}

1. Introduction. This paper deals with some reconstruction/restoration methods. These methods aim to recover a function defined on an open subset $\Omega$ of $\mathbb{R}^{2}$ from a sampled one, defined on $\Omega \cap \mathbb{Z}^{2}$. This latest data is assumed to be obtained itself by the composition of a convolution and a sampling applied on an unknown original function of $\Omega$. More precisely, given $\Omega$ an open set of $\mathbb{R}^{2}$, we have

$$
u_{m, n}=(s * v)(m, n)
$$

where $(m, n)$ belongs to $\Omega \cap \mathbb{Z}^{2}$, * denotes the continuous convolution, $s$ and $v$ map $\mathbb{R}^{2}$ onto $\mathbb{R}$, and $u$ maps $\Omega \cap \mathbb{Z}^{2}$ onto $\mathbb{R}$. Note that in this paper we will not take into account the edge effects in the vicinity of $\partial \Omega$; we will only focus on the cases where $\Omega=\mathbb{R}^{2}$, or $\Omega=(N \mathbb{T})^{2}$, the torus of size $N$.

Restoration and reconstruction are commonly distinguished in the fact that they respectively aim at recovering $v$ or $s * v$ on $\Omega$. Moreover, they both differ from interpolation, which aims at obtaining a "nice" function $w$, defined on $\Omega$, such that $w(m, n)=u_{m, n}$, for $(m, n) \in \Omega \cap \mathbb{Z}^{2}$. So, formally, this paper is concerned both with linear reconstructions (linear is used in the sense that the reconstruction of a sum of functions is the sum of the reconstructions) and some nonlinear restoration methods. However, since it is equivalent to linearly deconvolve a linear reconstruction or to directly expect a sharpest linear reconstruction, we do believe that these notions

* Received by the editor October 11, 1999; accepted for publication (in revised form) July 20, 2000; published electronically February 21, 2001.

http://www.siam.org/journals/sinum/39-1/36228.html

${ }^{\dagger}$ CMLA, ENS Cachan, 61 avenue du président Wilson, 94235 Cachan Cedex, France (malgouy@cmla.ens-cachan.fr, http://cmla.ens-cachan.fr/ malgouy). This author's work was partially financed by the French DGA.

${ }^{\ddagger}$ Poseidon Technologies, 3 rue nationale, 92100, France (fguichard@poseidon.fr, http://www. poseidon.fr). This author's work was supported by Cognitech, Inc. 
TABLE 1

Methods under our scope.

\begin{tabular}{|c|c|c|}
\hline & Section 2 & Section 3 \\
\hline Type of zooming & Reconstruction & Restoration \\
\hline Type of method & Linear & Nonlinear, maximum a posteriori \\
\hline Initial functional space & $l^{\infty}\left(\mathbb{Z}^{2}\right)$ & $N$-periodic elements of $l^{\infty}\left(\mathbb{Z}^{2}\right)$ \\
\hline Final functional space & $L^{\infty}\left(\mathbb{R}^{2}\right)$ & $L^{2}\left((N \mathbb{T})^{2}\right)$ \\
\hline
\end{tabular}

are similar (even if formally the restoration is a more general problem). Indeed, in practice, we just expect a "good" function of $\Omega$ from a sampled one.

However, we summarize in Table 1 the methods which fall under the scope of this paper. We remark that the use of $N$-periodic functions (with $N$ an integer) is equivalent to considering $\Omega=[0, N]^{2}$. Note also that some results similar to the one presented here are stated in [15], in the case of linear oversampling when the initial images are $N$-periodic and the result is defined over $(N \mathbb{T})^{2}$ (the torus of size $N$ ).

There have been several approaches in order to solve some problems of reconstruction/restoration. We can distinguish two main kinds: the linear and the nonlinear ones. Most of the linear methods aim to approximate the "sinc interpolation" (or "zero-padding") which consists in filling the lost part of the spectral domain with 0. This latest method is often considered as optimal because of the Shannon sampling theorem [24]. The shortcoming of this method is that the induced filter is badly localized in space domain (which yields a large algorithmic complexity for a linear interpolation) and oscillates. Some have reduced the algorithmic complexity (see [28]) in order to compute it, but most of the techniques propose to approximate it (see, for instance, $[19,26])$. Here, we will mathematically formalize in section 2 the framework of linear reconstructions, and we will show that most of the usual shortcomings of such methods cannot be simultaneously avoided. Consequently, it is not surprising to find numerous techniques to manage these shortcomings or that compare some linear reconstructions (see $[14,17]$ ).

Nonlinear methods generally take into account the local behavior of the initial function and adapt the reconstruction with regard to this behavior (see $[2,3,25]$ ). This essentially allows one to treat in a different manner the smooth zones and the vicinity of the edges inside the images. These reconstructions can yield good results but depend on the tool that determines the local behavior. Thus, they have several sources of shortcomings, and therefore, it is difficult to qualify their results. There also exist some variational techniques, the aim of which is to restore images. This will be commented on in section 3.3.

This paper is mainly concerned with the way the reconstruction/restoration methods under our scope deal with edges. More precisely, since edges arising in image processing are generally smooth (along the direction orthogonal to the one of gradient), they can be locally regarded as having a one-dimensional (1D) structure. (They are almost constant along the edge direction.) Therefore, we choose to model edges by what we call cylindric functions (those which are constant along a direction). We will see some conditions, which must satisfy the reconstruction/restoration methods under our scope, in order to preserve such functions. At last, we will validate this theoretical study by some experiments (see section 4 ).

This paper is organized as follows. In section 2 , we focus on linear reconstructions in the case $\Omega=\mathbb{R}^{2}$. Then we give a general property satisfied by such operators 
TABLE 2

Generic linear interpolation.

\begin{tabular}{|c|c|c|}
\hline Name & Form & Regularity \\
\hline Zero-crossing & $Z(u)=\sum_{m, n} u_{m, n} \delta_{m, n}$ & Measure \\
\hline Duplication & $Z(u)(x, y)=\sum_{m, n} u_{m, n} 1_{\left.[]-\frac{1}{2}, \frac{1}{2}\right]^{2}}(x-m, y-n)$ & Piecewise const. \\
\hline Zero-padding & $Z(u)(x, y)=\sum_{m, n} u_{m, n} \operatorname{sinc}_{2}(x-m, y-n)$ & $C^{\infty}$ \\
\hline
\end{tabular}

and define cylindric functions. Thereafter, we will prove that linear reconstructions that preserve cylindric images can be expressed in terms of a convolution of the sinc interpolation.

In section 3 , we deal with variational restoration methods, in the case where $\Omega=$ $\left[0, N \Gamma^{2}\right.$ with $N \in \mathbb{N}$. These methods minimize a convex "energy" $E$ among the function satisfying (1.1). Since we do believe the total variation is a good candidate to image restoration, we state our results in this particular case. Within this framework, we will show that the method is properly defined both theoretically and numerically. Then we exhibit some particular total variation based restorations that preserve cylindric images. The end of the section is devoted to other variational methods. This includes some argument in favor of the total variation and makes a link between some quadratic variational restorations and linear reconstructions.

Finally, some commented experiments are displayed in section 4.

2. Linear reconstructions and cylindric functions. In this section, we will characterize translation invariant linear reconstructions with regard to a geometric invariance property: the ability of a linear reconstruction to preserve images constant along a direction.

Let us introduce the framework and some notations that will be used throughout the section. We focus on the reconstruction of functions of $l^{\infty}\left(\mathbb{Z}^{2}\right)$ such that the reconstructed images do belong to $L^{\infty}\left(\mathbb{R}^{2}\right)$. With that in mind, we need to define $\hat{h}: \mathbb{R}^{2} \rightarrow \mathbb{R}$, the Fourier transform of $h \in L^{1}\left(\mathbb{R}^{2}\right)$, by

$$
\hat{h}(\xi, \eta)=\int_{\mathbb{R}^{2}} h(x, y) e^{-2 i \pi(\xi x+\eta y)} d x d y
$$

for $(\xi, \eta) \in \mathbb{R}^{2}$. Similarly, we also define the Fourier transform of a function $h \in l^{1}\left(\mathbb{Z}^{2}\right)$ by

$$
\hat{h}(\xi, \eta)=\sum_{(m, n) \in \mathbb{Z}^{2}} h_{m, n} e^{-2 i \pi(\xi m+\eta n)}
$$

for $\left.(\xi, \eta) \in]-\frac{1}{2}, \frac{1}{2}\right]^{2}$. One can refer to [16] for general properties of Fourier transforms.

We will also denote by $E^{*}$ the dual space of any linear space $E$ (see [23]).

Moreover, we define, in Table 2, the main generic linear interpolations, where $\delta_{m, n}$ stands for the Dirac delta function at the point $(m, n)$ and $\operatorname{sinc}_{2}\left(t_{1}, t_{2}\right)=$ $\operatorname{sinc}\left(\pi t_{1}\right) \operatorname{sinc}\left(\pi t_{2}\right)$ with $\operatorname{sinc}(t)=\frac{\sin (t)}{t}$ for $t \neq 0$ and $\operatorname{sinc}(0)=1$. Note that the zero-crossing is not really an interpolation since the result is not a function but a measure.

Let us first give some simple definitions and classical facts.

DeFinition 2.1. Let $Z$ be a linear operator continuous from $l^{\infty}\left(\mathbb{Z}^{2}\right)$ into $L^{\infty}\left(\mathbb{R}^{2}\right)$. $Z$ is called translation invariant if for any $u \in l^{\infty}\left(\mathbb{Z}^{2}\right)$, any $(m, n) \in \mathbb{Z}^{2}$, and any 
$(x, y) \in \mathbb{R}^{2}$,

$$
Z\left(T_{m, n}(u)\right)(x, y)=Z(u)(x-m, y-n),
$$

where $T_{m, n}$ denotes the translation of $(m, n)$ of a function of $l^{\infty}\left(\mathbb{Z}^{2}\right)\left(T_{m, n}(u)(k, l)=\right.$ $u(k-m, l-n))$.

We also define a weak notion of locality as follows.

DeFINITION 2.2. Let $Z$ be a linear operator continuous from $l^{\infty}\left(\mathbb{Z}^{2}\right)$ into $L^{\infty}\left(\mathbb{R}^{2}\right)$. Letting $u^{N}=u_{\mid\{-N, \ldots, N\}^{2}}$ the restriction of $u$ to $\{-N, \ldots, N\}^{2}$ for any $u \in l^{\infty}\left(\mathbb{Z}^{2}\right)$ and any $N \in \mathbb{N}$, we call $Z$ local if for any $u \in l^{\infty}\left(\mathbb{Z}^{2}\right)$ and any $(x, y) \in \mathbb{R}^{2}$, $\lim _{N \rightarrow \infty} Z\left(u^{N}\right)(x, y)$ exists and

$$
Z(u)(x, y)=\lim _{N \rightarrow \infty} Z\left(u^{N}\right)(x, y) .
$$

This notion avoids a linear operator that would not only depend on the local behavior of the function but also on its global one. Indeed, the Hahn-Banach theorem [29] permits us to extend an operator of the kind

$$
u \rightarrow Z(u)(x, y)=\lim _{|(m, n)| \rightarrow \infty} u_{m, n}
$$

(which is initially defined on $\left\{u \in l^{\infty}\left(\mathbb{Z}^{2}\right), \lim _{|(m, n)| \rightarrow \infty} u_{m, n}\right.$ exists $\}$ ) to $l^{\infty}\left(\mathbb{Z}^{2}\right)$. Such an operator is linear, translation invariant, and continuous from $l^{\infty}\left(\mathbb{Z}^{2}\right)$ into $L^{\infty}\left(\mathbb{R}^{2}\right)$ but does not reconstruct images in an interesting manner since the result is constant on $\mathbb{R}^{2}$. The above notion of locality ruled such operators out of our framework.

Linear operators that are both translation invariant and local can easily be characterized in terms of a convolution. More precisely, we have the following proposition.

Proposition 2.3. Let $Z$ be a linear, local, and translation invariant operator continuous from $l^{\infty}\left(\mathbb{Z}^{2}\right)$ into $L^{\infty}\left(\mathbb{R}^{2}\right)$. Then there exists a unique convolution kernel $h \in L^{1}\left(\mathbb{R}^{2}\right)$ and $C>0$ such that

$$
\forall(x, y) \in \mathbb{R}^{2}, \quad \sum_{(m, n) \in \mathbb{Z}^{2}}|h(x-m, y-n)|<C
$$

and

$$
\forall u \in l^{\infty}\left(\mathbb{Z}^{2}\right), \forall(x, y) \in \mathbb{R}^{2}, Z(u)(x, y)=\sum_{(m, n) \in \mathbb{Z}^{2}} u_{m, n} h(x-m, y-n) .
$$

The converse statement is also true.

Note that this result is close to classic ones (see [16, sections 2.1 and 3.2]). Its proof can be found in [15].

The operators described by this proposition are therefore some convolutions of the zero-crossing interpolation with a convolution kernel $h$. So it seems useless to describe local and translation invariant linear reconstructions in terms other than the ones related to this convolution kernel.

Also note that (2.1) implies that $h$ belongs to $L^{1}\left(\mathbb{R}^{2}\right)$. (So the assertion $h \in$ $L^{1}\left(\mathbb{R}^{2}\right)$ does not have any consequence on the above proposition.) Moreover, since $h \in L^{1}\left(\mathbb{R}^{2}\right)$, its Fourier transform exists and is a continuous function belonging to $L^{\infty}\left(\mathbb{R}^{2}\right)$. That is a point that will often be used in the rest of the section.

In the following, we will focus on a particular set of linear, local, and translation invariant operators, continuous from $l^{\infty}\left(\mathbb{Z}^{2}\right)$ to $L^{\infty}\left(\mathbb{R}^{2}\right)$, that are the regular ones. Regular will be used in the sense explained in the following definition. 
Definition 2.4. Let $Z$ be such as in Proposition 2.3. $Z$ is called regular if the kernel $h$ defining $Z$ satisfies

$$
\forall(\xi, \eta) \in \mathbb{R}^{2}, \quad \sum_{(k, l) \in \mathbb{Z}^{2}}|\hat{h}(\xi+k, \eta+l)|<C .
$$

This notion is clearly a notion of regularity. Indeed, functions satisfying (2.2) are continuous. Conversely, functions, the third derivatives of which are in $L^{1}\left(\mathbb{R}^{2}\right)$, satisfy $(2.2)$.

Under this assumption of regularity, the Poisson formula holds; in other words, we have the following lemma.

Lemma 2.5. Let $h \in L^{1}\left(\mathbb{R}^{2}\right)$ satisfy (2.1) and (2.2); then $h$ also satisfies

$$
\forall(\xi, \eta) \in \mathbb{R}^{2}, \quad \sum_{(m, n) \in \mathbb{Z}^{2}} h(m, n) e^{-2 i \pi(\xi m+\eta n)}=\sum_{(k, l) \in \mathbb{Z}^{2}} \hat{h}(\xi+k, \eta+l) .
$$

This result is classic (see $[16,30]$ ) and expresses the Fourier transform (the lefthand side term of (2.3)) of a sampling of a function according to the Fourier transform of the function.

Let us now state our definition of linear zooms.

DeFINITION 2.6. Let $Z$ be a linear, local, translation invariant, and regular operator continuous from $l^{\infty}\left(\mathbb{Z}^{2}\right)$ into $L^{\infty}\left(\mathbb{R}^{2}\right)$. $Z$ is called linear zoom if $Z$ transforms an image constant along an axis direction into an image constant along the same axis direction.

This definition of a linear zoom simply expresses the need for satisfying some basic properties such as a weak notion of locality, translation invariance (doing linear reconstruction, there are no reasons to a priori deal in different ways with the different parts of the initial image), regularity of the result, and continuity (which leads to the stability of the zoom), and also to impose them to restore correctly horizontal and vertical structures. Note also that this latter, in the case of signals, would be that the reconstruction of a constant signal is constant. Moreover, in such a case we could have stated a proposition similar to Proposition 2.7.

Now we are going to characterize these objects using $h$ (the kernel that defines $Z$ ).

Proposition 2.7. Let $Z$ be a linear, local translation invariant, and regular operator continuous from $l^{\infty}\left(\mathbb{Z}^{2}\right)$ into $L^{\infty}\left(\mathbb{R}^{2}\right)$. Let $h$ be the convolution kernel defining $Z$. $Z$ is a linear zoom if and only if

$$
\forall k \in \mathbb{Z} \backslash\{0\}, \forall \xi \in \mathbb{R}, \hat{h}(k, \xi)=\hat{h}(\xi, k)=0 .
$$

Proof. The proof is similar to the one of Proposition 2.9 below.

Note that we could as well have stated the conclusion of the proposition by saying that there exists $h$ such that

$$
\forall u \in l^{\infty}\left(\mathbb{Z}^{2}\right), Z(u)=h * u^{d},
$$

where $u^{d}$ denotes the duplication interpolation of the image $u$. Indeed, the Fourier transform of $1_{\left.[]-\frac{1}{2}, \frac{1}{2}\right]^{2}}$ satisfies $(2.4)$ and only vanishes on $\left\{(\xi, \eta) \in \mathbb{R}^{2}, \xi \in \mathbb{Z}\right.$, or $\eta \in \mathbb{Z}\}$.

Now we are going to state the definition of cylindric images. As we said in the introduction, these images have the property of fluctuating in only one direction. In 
the case of functions of $L^{\infty}\left(\mathbb{R}^{2}\right)$, we will simply define them as functions $u \in L^{\infty}\left(\mathbb{R}^{2}\right)$ such that there exists $(\alpha, \beta) \in \mathbb{R}^{2} \backslash\{(0,0)\}$ and $v \in L^{\infty}(\mathbb{R})$ satisfying

$$
u(x, y)=v(\alpha x+\beta y)
$$

for $(x, y) \in \mathbb{R}^{2}$.

Note that it is more difficult to define cylindric images of $l^{\infty}\left(\mathbb{Z}^{2}\right)$. Indeed, we remark that for any $u \in l^{\infty}\left(\mathbb{Z}^{2}\right)$ and any $(\alpha, \beta) \in \mathbb{R}^{2}$ such that the line $\alpha x+\beta y=0$ has an irrational slope, there exists a function $v \in L^{\infty}(\mathbb{R})$, such that

$$
\forall(m, n) \in \mathbb{Z}^{2}, u_{m, n}=v(\alpha m+\beta n) .
$$

This is simply due to the fact that all the $\alpha m+\beta n$ (with $m$ and $n$ in $\mathbb{Z}$ ) are distinct in $\mathbb{R}$.

So, defining cylindric functions of $l^{\infty}\left(\mathbb{Z}^{2}\right)$ as the ones that satisfy (2.5), we have to limit ourselves to functions $v$ such that the sampling does not create any aliasing. (We consider $u$ is obtained by a sampling of the function $V(x, y)=v(\alpha x+\beta y)$.) Therefore, we preserve the cylindric sight of the initial function. Consequently, the function $v$ in (2.5) has to be band-limited. More precisely, we use the following definition.

Definition 2.8. Let $u \in l^{\infty}\left(\mathbb{Z}^{2}\right)$. $u$ is called cylindric if and only if there exists a direction $(\alpha, \beta) \in \mathbb{R}^{2} \backslash\{(0,0)\}$ and $\tilde{v} \in\left(L^{\infty}\left(I_{\alpha, \beta}\right)\right)^{*}$ such that

$$
\forall(m, n) \in \mathbb{Z}^{2}, u_{m, n}=\int_{I_{\alpha, \beta}} \tilde{v}(\xi) e^{2 i \pi(\alpha m+\beta n) \xi} d \xi,
$$

where $I_{\alpha, \beta}$ is the largest interval such that $\forall \xi \in I_{\alpha, \beta},-\frac{1}{2}<\alpha \xi \leq \frac{1}{2}$ and $-\frac{1}{2}<\beta \xi \leq \frac{1}{2}$. Such an image will be called cylindric along the direction $(\alpha, \beta)$.

First, we remark that (2.6) should have been written using duality notation. However, we will abuse this notation even if it is formally valid only for $\tilde{v} \in L^{1}\left(I_{\alpha, \beta}\right)$.

Moreover, note that $(2.6)$ forces $u$ to belong to $l^{\infty}\left(\mathbb{Z}^{2}\right)$ and that, for any given $(\alpha, \beta)$, the definition of $I_{\alpha, \beta}$ ensures that $u$ satisfies (2.5) for the same couple $(\alpha, \beta)$ and a given function $v \in L^{\infty}(\mathbb{R})$.

Note also that, since Definition 2.8 is relatively restrictive, the sinc interpolation defined in the introduction gives an exact reconstruction for cylindric images (see [24]). We know, in other respects, that it yields oscillatory results (since the convolution kernel associated with the sinc interpolation oscillates). This latter behavior will not be taken into account by the analysis of cylindric functions.

Now let us state a proposition similar to Proposition 2.7 about linear zooms that preserve cylindric images. Note, once again, that the idea of being interested in the preservation of cylindric images is driven by the observation that a classical artifact in image reconstruction is the creation of staircase edges.

Proposition 2.9. Let $Z$ be a linear zoom and let $h$ be the convolution kernel that defines $Z$. Assume that for any direction $(\alpha, \beta) \in \mathbb{R}^{2} \backslash\{(0,0)\}$ and any image $u$ cylindric along the direction $(\alpha, \beta), Z(u)$ is also cylindric along the direction $(\alpha, \beta)$. Then

$$
\forall(\xi, \eta) \in \mathbb{R}^{2} \text { such that }|\xi|>\frac{1}{2} \text { or }|\eta|>\frac{1}{2}, \hat{h}(\xi, \eta)=0 .
$$

Conversely, any linear, local, and translation invariant operator continuous from $l^{\infty}\left(\mathbb{Z}^{2}\right)$ into $L^{\infty}\left(\mathbb{R}^{2}\right)$ associated with a kernel $h$ satisfying $(2.7)$ is a linear zoom and preserves cylindric images along any direction. 
Note that, heuristically, this proposition means that linear zooms preserving cylindric images can be expressed in terms of a convolution of the sinc interpolation. Unfortunately, we cannot give the proposition in this form since the sinc interpolation is not continuous from $l^{\infty}\left(\mathbb{Z}^{2}\right)$ into $L^{\infty}\left(\mathbb{R}^{2}\right)$.

In order to prove Proposition 2.9, we first state the following lemma.

Lemma 2.10. For any couple $(\alpha, \beta) \in \mathbb{R}^{2}$ such that the line defined by $\alpha x+\beta y=0$ has an irrational slope and for any $\left(a_{k, l}\right)_{k, l} \in l^{1}\left(\mathbb{Z}^{2}\right)$,

$$
\forall t \in \mathbb{R}, \quad \sum_{(k, l) \in \mathbb{Z}^{2}} a_{k, l} e^{-2 i \pi(\beta k-\alpha l) t}=0 \Longrightarrow \forall(k, l) \in \mathbb{Z}^{2}, a_{k, l}=0 .
$$

Proof. Note that for any $\left(a_{k, l}\right)_{k, l} \in l^{1}\left(\mathbb{Z}^{2}\right), \sum_{(k, l) \in \mathbb{Z}^{2}} a_{k, l} e^{-2 i \pi(\beta k-\alpha l) t}$ is in $L^{\infty}(\mathbb{R})$ and so it has a computable Fourier transform in the sense of tempered distributions. Therefore, the left part of (2.8) becomes

$$
\sum_{(k, l) \in \mathbb{Z}^{2}} a_{k, l} \delta_{(\beta k-\alpha l)}=0 .
$$

So, it is easy to check that, since the $(\beta k-\alpha l)$ are all distinct, this implies

$$
\forall(k, l) \in \mathbb{Z}^{2}, a_{k, l}=0 .
$$

Proof of Proposition 2.9. Let $(\alpha, \beta) \in \mathbb{R}^{2} \backslash\{(0,0)\}$ and let $u \in l^{\infty}\left(\mathbb{Z}^{2}\right)$ be a cylindric function along the direction $(\alpha, \beta)$. We know that there exists $\tilde{v} \in\left(L^{\infty}\left(I_{\alpha, \beta}\right)\right)^{*}$ linked with $u$ by $(2.6)$ and that there exists $h \in L^{1}\left(\mathbb{R}^{2}\right)$ such that

$$
Z(u)(x, y)=\sum_{(m, n) \in \mathbb{Z}^{2}} h(x-m, y-n) u_{m, n} .
$$

Using (2.6), (2.1), and the continuity of the operator defined by $\tilde{v}$ on $L^{\infty}\left(I_{\alpha, \beta}\right)$, we can write

$$
Z(u)(x, y)=\int_{I_{\alpha, \beta}} \tilde{v}(\xi) \sum_{(m, n) \in \mathbb{Z}^{2}} h(x-m, y-n) e^{2 i \pi(\alpha m+\beta n) \xi} d \xi .
$$

Then, if we write $h_{x, y}\left(x^{\prime}, y^{\prime}\right)=h\left(x+x^{\prime}, y+y^{\prime}\right)$, we have

$$
Z(u)(x, y)=\int_{I_{\alpha, \beta}} \tilde{v}(\xi) \sum_{(m, n) \in \mathbb{Z}^{2}} h_{x, y}(m, n) e^{-2 i \pi(\alpha m+\beta n) \xi} d \xi
$$

Using the Poisson formula (2.3), we get

$$
Z(u)(x, y)=\int_{I_{\alpha, \beta}} \tilde{v}(\xi) \sum_{(k, l) \in \mathbb{Z}^{2}} \widehat{h_{x, y}}(\alpha \xi+k, \beta \xi+l) d \xi .
$$

This formula expresses the fact that the spectrum of $Z(u)$ is the one of $u$, periodized and weighted by $h$ (see Figure 1 ).

Expressing the fact that $Z(u)$ is cylindric along the direction $(\alpha, \beta)$, we know that

$$
\forall t \in \mathbb{R}, Z(u)(x, y)-Z(u)(x-\beta t, y+\alpha t)=0,
$$




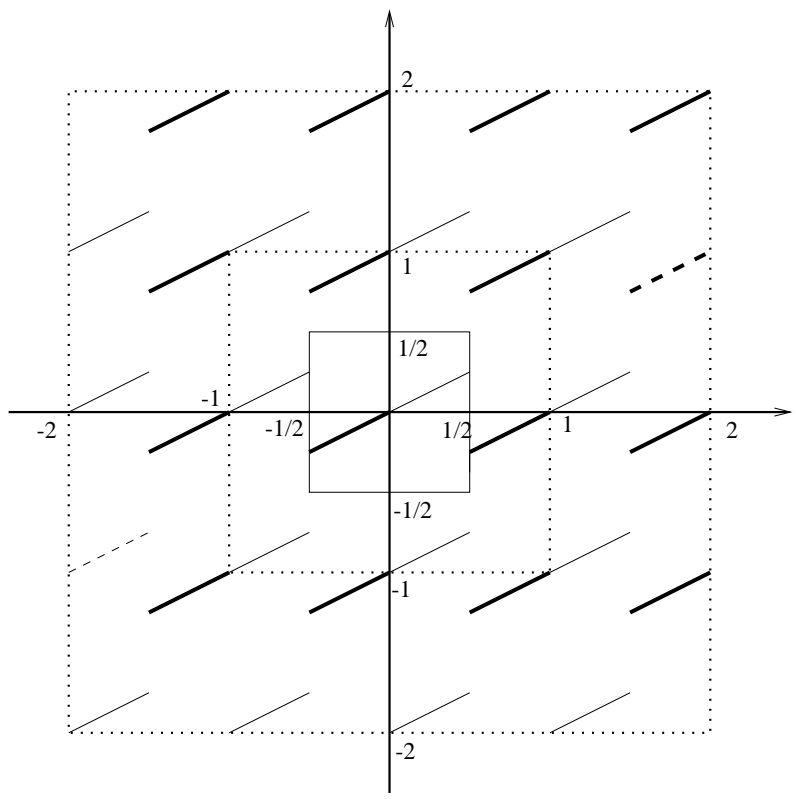

FIG. 1. The spectrum of the linear zoom is a weighted periodization of the initial image spectrum. This figure represents the support of a linear zoom of an image cylindric along the direction $(2,1)$. (The dashed segments are also in the direction $(2,1)$.)

which, using (2.9), gives

$$
\forall t \in \mathbb{R}, \int_{I_{\alpha, \beta}} \tilde{v}(\xi) \sum_{(k, l) \in \mathbb{Z}^{2}} \widehat{h_{x, y}}(\alpha \xi+k, \beta \xi+l)\left[1-e^{-2 i \pi(\beta k-\alpha l) t}\right] d \xi=0
$$

Since this equality holds for any $\tilde{v} \in\left(L^{\infty}\left(I_{\alpha, \beta}\right)\right)^{*}$, we finally have for any $t \in \mathbb{R}$

$$
\sum_{(k, l) \in \mathbb{Z}^{2}} \widehat{h_{x, y}}(\alpha \xi+k, \beta \xi+l) e^{-2 i \pi(\beta k-\alpha l) t}=\sum_{(k, l) \in \mathbb{Z}^{2}} \widehat{h_{x, y}}(\alpha \xi+k, \beta \xi+l) .
$$

Therefore, using Lemma 2.10, we finally find that if $Z$ preserves cylindric functions along any direction, $\hat{h}(\xi, \eta)=0$ for any $(\xi, \eta) \in \mathcal{C}$ with

$$
\mathcal{C}=\underset{(k, l) \neq(0,0)}{\cup} \mathcal{C}_{k, l}
$$

and

$$
\begin{array}{r}
\mathcal{C}_{k, l}=\left\{(\alpha \xi+k, \beta \xi+l),(\alpha, \beta) \in \mathbb{R}^{2} \backslash\{(0,0)\}\right. \text { such that the line } \\
\left.-\beta y+\alpha x=0 \text { has an irrational slope and } \xi \in I_{\alpha, \beta}\right\} .
\end{array}
$$

It is easy to check that $\mathcal{C}_{k, l}$ is dense in $\left.\left.\left.] k-\frac{1}{2}, k+\frac{1}{2}\right] \times\right] l-\frac{1}{2}, l+\frac{1}{2}\right]$ and that $\mathcal{C}$ is dense in $\left.\mathbb{R}^{2} \backslash\right]-\frac{1}{2}, \frac{1}{2}\left[^{2}\right.$.

The result follows from the fact that (2.1) imposes $\hat{h}$ to be continuous.

The converse statement directly follows from (2.9). 
We remark that in this proof we could have avoided the use of $(\alpha, \beta)$ such that $\alpha x+\beta y=0$ has an irrational slope. Indeed, going a little bit further in the analysis (see Figure 1), we could have obtained

$$
\begin{aligned}
\mathcal{C}_{k, l}=\{(\alpha \xi+k, \beta \xi+l), & (\alpha, \beta) \in \mathbb{R}^{2} \backslash\{(0,0)\} \\
& \text { such that } \left.-\beta k+\alpha l \neq 0 \text { and } \xi \in I_{\alpha, \beta}\right\} .
\end{aligned}
$$

Note that even the restriction of this $\mathcal{C}_{k, l}$ to the couples $(\alpha, \beta)$, such that $\alpha x+\beta y=0$ has a rational slope, is dense in $\left[k-\frac{1}{2}, k+\frac{1}{2}\right] \times\left[l-\frac{1}{2}, l+\frac{1}{2}\right]$.

At this point, in order to determine an $h$ yielding good results, the most important remaining problem is the locality of $h$. (Otherwise we have blurred and/or oscillating results and large computation cost.) Note that, since $\hat{h}$ is compactly supported, we can neither expect $h$ to be well located nor to yield sharp results. However, we can suggest to readers that they learn about prolate functions [13], which are a way to deal with the Heisenberg uncertainty principle (see [16]). Unfortunately, such functions define reconstructions which lead to blurred results (see section 4).

Now, this analysis shows us that linear reconstructions cannot yield fully satisfactory results, in the sense that they cannot completely avoid all the artifacts mentioned above. This is simply due to the fact that the erasure of these artifacts yields to contradictory properties of the kernel $h$. Therefore, if one still wants to reconstruct images linearly, one has to balance all these shortcomings with regard to the expected properties the reconstructed images shall satisfy.

3. Variational image restorations. In this section we will focus on possibly nonlinear restorations. Formally, we have to talk about restoration since we do take into account the whole degradation suffered by the image (including the convolution).

Let us introduce the framework and some notations that will be used throughout the section.

First, these restorations are based on functional minimization, so we turn to a finite dimensional case in order to get proper results. Therefore, a sampled image $u$ is assumed to be periodic of period $N$. (For commodity we will assume in the following that $N$ is even.) The discrete Fourier transform (DFT) of such a $u$ is defined for any $(\xi, \eta) \in\left\{-\frac{N}{2}+1, \ldots, \frac{N}{2}\right\}^{2}$ by

$$
\hat{u}_{\xi, \eta}=\sum_{m, n=0}^{N-1} u_{m, n} e^{-2 i \pi \frac{\xi m+\eta n}{N}} .
$$

Since the sampled images are periodic of period $N$, we define the zoomed images on the torus of size $N,(N \mathbb{T})^{2}$. (But by an abuse of the language we will note it $\mathbb{T}^{2}$ ). In addition and despite a lack of generality we are able only to state results in the space $L^{2}\left(\mathbb{T}^{2}\right)$ (and not $L^{\infty}\left(\mathbb{T}^{2}\right)$ ). However, we have not found any case (neither theoretical nor numerical) where the sup-norm of the result tends to infinity. The Fourier series of an image $w \in L^{2}\left(\mathbb{T}^{2}\right)$ is defined on $\left(\frac{1}{N} \mathbb{Z}\right)^{2}$ by

$$
\hat{w}_{\frac{k}{N}, \frac{l}{N}}=\int_{\mathbb{T}^{2}} w(x, y) e^{-2 i \pi \frac{(k x+l y)}{N}}
$$

for $(k, l) \in \mathbb{Z}^{2}$. The inverse Fourier transform, in such a case, is defined by

$$
w(x, y)=\frac{1}{N^{2}} \sum_{(k, l) \in \mathbb{Z}^{2}} \hat{w}_{\frac{k}{N}, \frac{l}{N}} e^{2 i \pi \frac{(k x+l y)}{N}}
$$

for $(x, y) \in \mathbb{T}^{2}$. 
In the following, in order to distinguish Fourier series from DFTs, we will denote $\hat{w}_{\frac{k}{N}, \frac{l}{N}}$ by $\hat{w}\left(\frac{k}{N}, \frac{l}{N}\right)$ in the case of the Fourier series. Moreover, the frequency coordinates will often be written $\left(\frac{\xi}{N}+k, \frac{\eta}{N}+l\right)$ with $(\xi, \eta) \in\left\{-\frac{N}{2}+1, \ldots, \frac{N}{2}\right\}^{2}$ and $(k, l) \in \mathbb{Z}^{2}$ to give prominence to the frequency on which it aliases.

Moreover, we present the method in the case of a regularity criterion that is the total variation, but most of the results can be extended to other kinds of energies that would satisfy properties such as "lower semicontinuity" and "compactness" and which satisfy

$$
E(h * w) \leq\|h\|_{1} E(w)
$$

for $h \in L^{1}\left(\mathbb{T}^{2}\right)$. However, we present this work in the case of the total variation since we do believe it is the most suitable for image reconstruction (see section 3.3.2).

Now let us present the method under study and the associated results.

3.1. Total variation based restoration. As far as we know, the use of the total variation in image processing was first introduced in [21]. It has since been studied by several authors and under several points of view in [1, 4, 6, 7, 8, 11, 18, 22]. To our knowledge, its use in image oversampling was first introduced in [10]. Here, the idea is to apply a maximum a posteriori regularization approach to the problem of image zooming. With that in mind, we first have to define the set of all possible images of $L^{2}\left(\mathbb{T}^{2}\right)$ that are sampled in the discrete $N$-periodic image $u \in l^{\infty}\left(\mathbb{Z}^{2}\right)$.

As we said in the introduction, we assume the sampled image $u$ is deduced from a continuous one $v$ by the composition of a convolution and a multiplication by a Dirac comb. So, the set of all the candidates to be the result of the restoration is defined by the following.

DeFinition 3.1. Let $N$ be a strictly positive integer, let $u \in l^{\infty}\left(\mathbb{Z}^{2}\right)$ be an $N$ periodic function, and let $s \in L^{2}\left(\mathbb{T}^{2}\right)$. We define $\mathcal{W}_{s, u}$, the set of all the functions $w \in L^{2}\left(\mathbb{T}^{2}\right)$ that satisfy

$$
\forall(m, n) \in\{0, \ldots, N-1\}^{2}, u_{m, n}=(s * w)(m, n) .
$$

In other terms, $\mathcal{W}_{s, u}$ is the set of the images which yield $u$ after a convolution with $s$ and a sampling.

In this definition, the smoothing $s$ can be arbitrary, but in practice we mainly focus on two simple kernels yielding good properties when used to restore $N$-periodic cylindric functions (in axis or in any direction). These are the "mean kernel"

$$
s^{m}(x, y)=1_{\left.[]-\frac{1}{2}, \frac{1}{2}\right]^{2}}(x, y)
$$

for $(x, y) \in \mathbb{T}^{2}$ and the "frequency extrapolation kernel" (or "sinc") $s^{e}$ defined by its Fourier series

$$
\widehat{s^{e}}\left(\frac{k}{N}, \frac{l}{N}\right)=1_{\mid\left\{-\frac{1}{2}+\frac{1}{N}, \ldots, \frac{1}{2}\right\}^{2}}\left(\frac{k}{N}, \frac{l}{N}\right)
$$

for $(k, l) \in \mathbb{Z}^{2}$. (Note that $s^{e}$ belongs to $L^{\infty}\left(\mathbb{T}^{2}\right)$ and, consequently, $L^{2}\left(\mathbb{T}^{2}\right)$.) In this latter case, frequencies of $w$ higher than $\frac{1}{2}$ in modulus are simply set free by the constraint (3.1) (see (3.4) below).

We remark that, since the convolution and the sampling operator are linear, for any convolution kernel $s, \mathcal{W}_{s, u}$ is an affine subspace of $L^{2}\left(\mathbb{T}^{2}\right)$. 
In order to interpret the degrees of freedom we have inside $\mathcal{W}_{s, u}$, we express (3.1) in frequency domain. A Poisson formula (similar to (2.3) but adapted to periodic functions) yields

$$
\hat{u}_{\xi, \eta}=\sum_{(k, l) \in \mathbb{Z}^{2}} \hat{s}\left(\frac{\xi}{N}+k, \frac{\eta}{N}+l\right) \widehat{w}\left(\frac{\xi}{N}+k, \frac{\eta}{N}+l\right),
$$

where $(\xi, \eta) \in\{-N / 2+1, \ldots, N / 2\}^{2}$.

Looking at (3.4), we see that the degrees of freedom mentioned above are simply the repartition of $\hat{u}_{\xi, \eta}$ onto $\left(\widehat{w}\left(\frac{\xi}{N}+k, \frac{\eta}{N}+l\right)\right)_{k, l}$. Moreover, (3.4) gives us a sufficient condition for $\mathcal{W}_{s, u}$ not to be empty. In the following we will assume that $\hat{s}$ does not vanish on $\left\{-\frac{1}{2}+\frac{1}{N}, \ldots, \frac{1}{2}\right\}^{2}$, so that for any $N$-periodic $u \in l^{\infty}\left(\mathbb{Z}^{2}\right)$ the function $u^{c}$ defined by

$$
\widehat{u^{c}}\left(\frac{\xi}{N}+k, \frac{\eta}{N}+l\right)=\left\{\begin{array}{cc}
\frac{\hat{u}_{\xi, \eta}}{\hat{s}\left(\frac{\xi}{N}, \frac{\eta}{N}\right)} & \text { if }(k, l)=(0,0), \\
0 & \text { otherwise }
\end{array}\right.
$$

for $(\xi, \eta) \in\{-N / 2+1, \ldots, N / 2\}^{2}$ and $(k, l) \in \mathbb{Z}^{2}$ belongs to $\mathcal{W}_{s, u}$.

At this point, we have to select an element of $\mathcal{W}_{s, u}$, given $u$ and $s$. We propose here to choose the one that is the most "regular," in the sense that its total variation is minimal, say,

$$
Z(u)=\operatorname{argmin}\left(|D w|\left(\mathbb{T}^{2}\right)\right), \text { among } w \in \mathcal{W}_{s, u} .
$$

One can refer to [9] for general results on total variation. We just recall that

$|D w|\left(\mathbb{T}^{2}\right)=\sup \left\{\int_{\mathbb{T}^{2}} w \operatorname{div}(\varphi), \varphi \in C^{1}\left(\mathbb{T}^{2}, \mathbb{R}^{2}\right)\right.$ compactly supported and $\left.\|\varphi\|_{\infty} \leq 1\right\}$

and that it is a seminorm equal to $\int_{\mathbb{T}^{2}}|\nabla w|$, when $w$ is $C^{1}$. Moreover, we will call $B V\left(\mathbb{T}^{2}\right)$ the space of functions belonging to $L^{1}\left(\mathbb{T}^{2}\right)$ and having a finite total variation, and the associated norm is $\|\cdot\|_{B V}=\|\cdot\|_{1}+|D \cdot|\left(\mathbb{T}^{2}\right)$.

3.1.1. Existence and uniqueness of a solution. Let us state the proposition ensuring the existence of a solution to (3.6).

Proposition 3.2. Let $N \in \mathbb{N}, u \in l^{\infty}\left(\mathbb{Z}^{2}\right) N$-periodic, and let $s \in L^{2}\left(\mathbb{T}^{2}\right)$ such that $\hat{s}$ does not vanish on $\left\{-\frac{1}{2}+\frac{1}{N}, \ldots, \frac{1}{2}\right\}^{2}$ and $\hat{s}(k, l)=0$ for any $(k, l) \in \mathbb{Z}^{2} \backslash\{(0,0)\}$; then (3.6) admits a solution $v \in \mathcal{W}_{s, u} \cap B V\left(\mathbb{T}^{2}\right)$.

Note that, looking at $(3.4), \hat{s}(k, l)=\hat{s}\left(\frac{0}{N}+k, \frac{0}{N}+l\right)=0$, for any $(k, l) \in$ $\mathbb{Z}^{2} \backslash\{(0,0)\}$, simply forces functions of $\mathcal{W}_{s, u}$ to have the same mean.

Proof. The following proof is similar to the one presented in [6] in the deblurring case. Let $v_{n}$ be a sequence minimizing the total variation and satisfying the constraint. (Note that such a sequence exists since (3.5) defines a function of $\mathcal{W}_{s, u}$ which has a finite total variation.) We remark that, since $v_{n} \in \mathcal{W}_{s, u}$ and $\mathbb{T}^{2}$ is bounded, $v_{n}$ is in $L^{1}\left(\mathbb{T}^{2}\right)$ and therefore in $B V\left(\mathbb{T}^{2}\right)$. So, Sobolev inequalities (see [9, Theorem 1, p. 189] ensure that there exists $C>0$

$$
\left\|v_{n}\right\|_{2} \leq C\left|D v_{n}\right|\left(\mathbb{T}^{2}\right)+N \int_{\mathbb{T}^{2}} v_{n}
$$


So, since $\int_{\mathbb{T}^{2}} v_{n}$ is fixed, $\left\|v_{n}\right\|_{2},\left\|v_{n}\right\|_{1}$, and $\left\|v_{n}\right\|_{B V}$ are bounded. Therefore, compactness theorems both in $B V\left(\mathbb{T}^{2}\right)$ and $L^{2}\left(\mathbb{T}^{2}\right)$ (see [9]) yield that there exists a subsequence $v_{n_{j}}$ that converges in $L^{1}\left(\mathbb{T}^{2}\right)$ and weakly in $L^{2}\left(\mathbb{T}^{2}\right)$ to a function $v \in$ $B V\left(\mathbb{T}^{2}\right) \cap L^{2}\left(\mathbb{T}^{2}\right)$.

It is easy to check that $v$ is a solution of (3.6). Indeed, the weak convergence in $L^{2}$ of $v_{n_{j}}$ ensures that $v \in \mathcal{W}_{s, u}$, and the lower semicontinuity of the total variation ensures that $|D v|\left(\mathbb{T}^{2}\right) \leq \liminf _{j \rightarrow \infty}\left|D v_{n_{j}}\right|\left(\mathbb{T}^{2}\right)$.

The "sinc" function $s^{e}$ obviously falls under the scope of the above proposition. Concerning $s^{m}$, we have for any $(k, l) \in \mathbb{Z}^{2}$

$$
\widehat{s^{m}}(k / N, l / N)=\operatorname{sinc}(\pi k / N) \operatorname{sinc}(\pi l / N)
$$

with $\operatorname{sinc}(x)=\frac{\sin (x)}{x}$ if $x \neq 0$ and 1 otherwise. So $s^{m}$ satisfies the hypotheses of the above proposition. Moreover, we remark that for any $h \in L^{2}\left(\mathbb{T}^{2}\right)$ such that $\hat{h}$ does not vanish on $\left\{-\frac{1}{2}+\frac{1}{N}, \ldots, \frac{1}{2}\right\}^{2}, h * s^{e}$ and $h * s^{m}$ also satisfy the hypotheses of Proposition 3.2 .

We are not necessarily sure of the uniqueness of this solution, since the total variation is not strictly convex. Nevertheless, we can see that minimizers are "close" to each other in the sense that for almost every point, where their gradients exist and are not null, they have the same gradient direction, as explained in [8].

Proposition 3.3 ("Weak Uniqueness"). Let $v_{1}$ and $v_{2}$ be two solutions of the problem (3.6). If we note

$$
\Gamma=\left\{(x, y) \in \mathbb{T}^{2}, v_{1} \text { and } v_{2} \text { are } C^{1} \text { at }(x, y) \text { and } \nabla v_{1}(x, y) \neq 0\right\}
$$

for any $(x, y) \in \Gamma$, there exists $\lambda \geq 0$ such that

$$
\nabla v_{2}(x, y)=\lambda \nabla v_{1}(x, y)
$$

Note that if $v_{1}$ and $v_{2}$ are both continuously differentiable on $\mathbb{T}^{2}$, then this proposition means that the connected components (in $\left\{(x, y) \in \mathbb{T}^{2}, \nabla v_{1}(x, y) \neq\right.$ 0 and $\left.\left.\nabla v_{2}(x, y) \neq 0\right\}\right)$ of the level lines of $v_{1}$ and $v_{2}$ are identical. Thus, the two minima somehow differ in a local change of contrast. (One can refer to [5] for an example of definition of contrast change related to level sets.)

3.1.2. Total variation based restoration and cylindric functions. We are now going to state two propositions which show the behavior of the total variation based restoration when dealing with cylindric functions. This leads us to restrict the set of cylindric functions (defined in Definition 2.8) to the ones that are of period $N$. Therefore, taking notations of the Definition 2.8, for any direction $(\alpha, \beta) \neq(0,0)$, any $(m, n) \in \mathbb{Z}^{2}$, and any $\left(t, t^{\prime}\right) \in \mathbb{Z}^{2}$,

$$
\int_{I_{\alpha, \beta}} \tilde{v}(\xi) e^{2 i \pi(\alpha m+\beta n) \xi} d \xi=\int_{I_{\alpha, \beta}} \tilde{v}(\xi) e^{2 i \pi(\alpha m+\beta n) \xi} e^{2 i \pi\left(\alpha t N+\beta t^{\prime} N\right) \xi} d \xi .
$$

So, since this equality holds for $(m, n) \in \mathbb{Z}^{2}$, the $\tilde{v}$ measure of $\left\{\xi, e^{2 i \pi\left(\alpha t N+\beta t^{\prime} N\right) \xi} \neq 1\right\}$ is 0 . Thus, $\alpha N \xi$ and $\beta N \xi$ must belong to $\mathbb{Z}((\alpha, \beta)$ must be chosen such that the line $\alpha x+\beta y=0$ has a rational slope) and $\tilde{v}$ must be a sum of Dirac delta functions. More precisely, we have

$$
\forall(m, n) \in \mathbb{Z}^{2}, u_{m, n}=\sum_{\xi \in \tilde{I}_{\alpha, \beta}} \tilde{v}(\xi) e^{2 i \pi(\alpha m+\beta n) \xi},
$$


where $\tilde{I}_{\alpha, \beta}$ is a discrete set such that $\forall \xi \in \tilde{I}_{\alpha, \beta}$, there exists $(k, l) \in \mathbb{Z}^{2}$ such that $\alpha N \xi=k$ and $\beta N \xi=l$ with $-\frac{1}{2} \leq \alpha \xi \leq \frac{1}{2}$ and $-\frac{1}{2} \leq \beta \xi \leq \frac{1}{2}$. Note that, in such a case, the summation in (3.7) can be done over $(k, l) \in\{-N / 2+1, \ldots, N / 2\}^{2}$ satisfying $\beta k-\alpha l=0$. This means that the DFT of $u$ is supported on the trail of the line $\beta x-\alpha y=0$ on the grid $\left\{\left(\frac{k}{N}, \frac{l}{N}\right),-N / 2+1 \leq k, l \leq N / 2\right\}$. Therefore, we state the following definition.

Definition 3.4. Let $u \in l^{\infty}\left(\mathbb{Z}^{2}\right)$ be periodic of period $N$ and $(\alpha, \beta) \in \mathbb{Z}^{2} \backslash\{(0,0)\}$. $u$ is cylindric along the direction $(\alpha, \beta)$ if and only if its DFT is supported on

$$
\left\{(k, l) \in\left\{-\frac{N}{2}+1, \ldots, \frac{N}{2}\right\}^{2}, \beta k-\alpha l=0\right\} .
$$

We remark that the larger $N$ is, the less the restriction to periodic images is harmful to the meaning of the results dealing with $N$-periodic cylindric functions.

The two following propositions give sufficient conditions on $s$ so that (3.6) defines a zoom that preserves cylindric functions. To do so, we construct a cylindric solution $v^{\prime}$ given a solution $v$. Unfortunately, we cannot guarantee all the solutions of (3.6) to be cylindric.

Proposition 3.5. Let $N$ be an integer, $u \in l^{\infty}\left(\mathbb{Z}^{2}\right) N$-periodic and cylindric along an axis direction. For any kernel $s=h * s^{m}$ (see (3.2)), with $h \in L^{2}\left(\mathbb{T}^{2}\right)$ such that $\hat{h}$ does not vanish on $\left\{-\frac{1}{2}+\frac{1}{N}, \ldots, \frac{1}{2}\right\}^{2},(3.6)$ admits a solution cylindric along the same axis direction.

Proof. The proof of this proposition is similar to the one of the next proposition.

Proposition 3.6. Let $N$ be an integer, $(\alpha, \beta) \in \mathbb{R}^{2} \backslash\{(0,0)\}, u \in l^{\infty}\left(\mathbb{Z}^{2}\right) N$ periodic and cylindric along the direction $(\alpha, \beta)$. For any kernel $s=h * s^{e}$ (see (3.3)), with $h \in L^{2}\left(\mathbb{T}^{2}\right)$ such that $\hat{h}$ does not vanish on $\left\{-\frac{1}{2}+\frac{1}{N}, \ldots, \frac{1}{2}\right\}^{2}$, (3.6) admits a solution cylindric along the same direction $(\alpha, \beta)$.

Proof. Let $v \in \mathcal{W}_{s, u}$ a solution of (3.6). (We know one exists since $s=h * s^{e}$ satisfies the hypotheses of Proposition 3.2.) Letting $M \in \mathbb{R}$, we define the function

$$
v_{M}(x, y)=\frac{1}{2 M} \int_{-M}^{M} v(x-\beta t, y+\alpha t) d t
$$

for $(x, y) \in \mathbb{T}^{2}$.

Now we are going to show that the limit of $v_{M}$, when $M$ tends to infinity, exists, is a solution of (3.6), and is cylindric along the right direction. In order to do this, let us first estimate $\left|D v_{M}\right|\left(\mathbb{T}^{2}\right)$. We have for any $\varphi \in C^{1}\left(\mathbb{T}^{2}, \mathbb{R}^{2}\right)$ compactly supported, such that $\|\varphi\|_{\infty} \leq 1$ and any $M>0$,

$$
\begin{aligned}
\int_{\mathbb{T}^{2}} v_{M}(x, y) \operatorname{div} \varphi(x, y) d x d y & =\frac{1}{2 M} \int_{\mathbb{T}^{2}} \int_{-M}^{M} v(x-\beta t, y+\alpha t) \operatorname{div} \varphi(x, y) d t d x d y \\
& =\frac{1}{2 M} \int_{-M}^{M} \int_{\mathbb{T}^{2}} v(x-\beta t, y+\alpha t) \operatorname{div} \varphi(x, y) d x d y d t \\
& \leq \frac{1}{2 M} \int_{-M}^{M}|D v|\left(\mathbb{T}^{2}\right) d t \\
& \leq|D v|\left(\mathbb{T}^{2}\right) .
\end{aligned}
$$

So, for any $M>0$,

$$
\left|D v_{M}\right|\left(\mathbb{T}^{2}\right) \leq|D v|\left(\mathbb{T}^{2}\right) .
$$


Therefore, just as in the proof of Proposition 3.2, Sobolev inequalities, combined with the fact that for any $M, v_{M}$ has the same mean, ensure that $\left\|v_{M}\right\|_{2}$ and $\left\|v_{M}\right\|_{B V}$ are bounded. Thus, we can extract a subsequence $\left(v_{M_{j}}\right)_{j \in \mathbb{N}}$ that converges in $L^{1}$, to a function $v^{\prime} \in B V\left(\mathbb{T}^{2}\right) \cap L^{2}\left(\mathbb{T}^{2}\right)$ which satisfies

$$
\left|D v^{\prime}\right|\left(\mathbb{T}^{2}\right) \leq \liminf _{j \rightarrow \infty}\left|D v_{M_{j}}\right|\left(\mathbb{T}^{2}\right) \leq|D v|\left(\mathbb{T}^{2}\right) .
$$

In order to show that $v^{\prime}$ is a solution of (3.6), we still have to prove that $v^{\prime}$ belongs to $\mathcal{W}_{s, u}$. We are going to show, at the same time, that $v^{\prime}$ is cylindric of direction $(\alpha, \beta)$. Therefore, we compute for $j \in \mathbb{N}$ and $(k, l) \in \mathbb{Z}^{2}$,

$$
\begin{aligned}
\widehat{v_{M_{j}}}\left(\frac{k}{N}, \frac{l}{N}\right) & =\hat{v}\left(\frac{k}{N}, \frac{l}{N}\right) \frac{1}{2 M_{j}} \int_{-M_{j}}^{M_{j}} e^{-2 i \pi\left(\beta \frac{k}{N}-\alpha \frac{l}{N}\right) t} d t \\
& =\hat{v}\left(\frac{k}{N}, \frac{l}{N}\right) \operatorname{sinc}\left(2 \pi \frac{\beta k-\alpha l}{N} M_{j}\right) .
\end{aligned}
$$

Thus, since $v_{M_{j}}$ converges in $L^{1}$ to $v^{\prime}$ (and so $\widehat{v_{M_{j}}}$ converges in $l^{\infty}$ to $\widehat{v^{\prime}}$ ), we have for any $(k, l) \in \mathbb{Z}^{2}$ such that $\beta k-\alpha l=0$

$$
\widehat{v^{\prime}}\left(\frac{k}{N}, \frac{l}{N}\right)=\lim _{j \rightarrow \infty} \widehat{v_{M_{j}}}\left(\frac{k}{N}, \frac{l}{N}\right)=\hat{v}\left(\frac{k}{N}, \frac{l}{N}\right)
$$

and for those such that $\beta k-\alpha l \neq 0$

$$
\widehat{v^{\prime}}\left(\frac{k}{N}, \frac{l}{N}\right)=\lim _{j \rightarrow \infty} \widehat{v_{M_{j}}}\left(\frac{k}{N}, \frac{l}{N}\right)=0 .
$$

So $v^{\prime}$ is cylindric along the direction $(\alpha, \beta)$. Moreover, since $v$ belongs to $\mathcal{W}_{s, u}$ and $u$ is cylindric along the direction $(\alpha, \beta)$, we are sure that $v^{\prime} \in \mathcal{W}_{s, u}$.

We remark that the advantage of the total variation based restoration over linear reconstructions (that preserves cylindric functions) is that it allows us to extrapolate the frequencies out of $\left\{-\frac{1}{2}+\frac{1}{N}, \ldots, \frac{1}{2}\right\}^{2}$. Therefore, here we do not a priori have to choose between "ringing" and "blurring" artifacts (see section 4).

Also note that in the proof of this proposition we have used only the form of the constraint and the fact that the total variation decreases after a convolution with a function $h$ such that $\int_{\mathbb{T}^{2}}|h|=1$. So, such a proposition can be obtained doing the same reasoning for other kind of energies such as, for instance, $\int_{\mathbb{T}^{2}}|L(w)|^{r}$, where $L$ is a differential operator.

3.2. Numerical approximation of the total variation based restoration. Let us first show two consistency propositions which ensure that a solution of (3.6) can be approximated numerically. With that in mind, we first define a problem, the solutions of which are computable and approximate a solution of (3.6).

Let $N, u, s$ be such as in Proposition 3.2 and let $K>1$ be an integer; we define $\mathcal{W}_{s, u}^{K}$ by

$$
w \in \mathcal{W}_{s, u}^{K} \Longleftrightarrow\left\{\begin{array}{l}
w \in \mathcal{W}_{s, u} \text { and } \\
\forall(k, l) \in \mathbb{Z}^{2} \backslash\left\{-\frac{K N}{2}+1, \ldots, \frac{K N}{2}-1\right\}^{2}, \widehat{w}\left(\frac{k}{N}, \frac{l}{N}\right)=0 .
\end{array}\right.
$$

Since $\mathcal{W}_{s, u}^{K}$ is a finite dimensional affine space, its elements can be manipulated numerically. Therefore, we define the band-limited approximating restoration by

$$
v^{K}=\operatorname{argmin}\left(|D w|\left(\mathbb{T}^{2}\right)\right), \text { among } w \in \mathcal{W}_{s, u}^{K} .
$$


Note that such a minimum exists since $u^{c} \in \mathcal{W}_{s, u}^{K}$ (see (3.5)) and since we minimize a coercive and convex functional on $\mathcal{W}_{s, u}^{K}$ (a finite dimensional affine space). Note also that since functions of $\mathcal{W}_{s, u}^{K}$ are $C^{1}$ we have $|D w|\left(\mathbb{T}^{2}\right)=\int_{\mathbb{T}^{2}}|\nabla w|$ for any $w \in$ $\mathcal{W}_{s, u}^{K}$. For any given $K>1$, the associated band-limited approximating restoration represents a zoom of factor $K$ (in the sense that the image resolution is increased by the factor $K$ ). Proposition 3.8 will prove that $v^{K}$ can be used for $K$ large to evaluate a solution of (3.6).

Unfortunately, we are not able to compute $\int_{\mathbb{T}^{2}}|\nabla w|$ (and so we cannot numerically minimize it), even for an image in $\mathcal{W}_{s, u}^{K}$. The problem is that even when $|\nabla w|$ is defined everywhere (functions of $\mathcal{W}_{s, u}^{K}$ are band-limited), we cannot integrate it on the torus. So, we estimate the integral with a Riemann summation and approximate the total variation by

$$
E_{K^{\prime}}(w)=\frac{1}{K^{\prime 2}} \sum_{m, n=0}^{K^{\prime} N-1}\left|\nabla w\left(\frac{m}{K^{\prime}}, \frac{n}{K^{\prime}}\right)\right|
$$

We define the solvable restoration (the solution of which are numerically computable) by

$$
v^{K, K^{\prime}}=\operatorname{argmin}\left(E_{K^{\prime}}(w)\right), \text { among } w \in \mathcal{W}_{s, u}^{K} .
$$

Note once again that $v^{K, K^{\prime}}$ exists and that it can be estimated by a method similar to the one described in $[7,11]$. (Note that in [7] and [11] the image degradation is inclusive of noise.)

The following proposition shows that a solution $v^{K}$ of the band-limited approximating restoration (3.10) can be approximated by the sequence $\left(v^{K, K^{\prime}}\right)_{K^{\prime} \geq K}$ when $K^{\prime}$ grows to infinity. One can refer to [4] where the authors boil down to a computable problem by a restriction to piecewise constant functions, in the case of image deblurring.

Proposition 3.7. Let $N$ and $K$ be two integers (with $K>1$ ), $u \in l^{\infty}\left(\mathbb{Z}^{2}\right.$ ) $N$-periodic, and $s \in L^{2}\left(\mathbb{T}^{2}\right)$ satisfying the hypotheses of the Proposition 3.2. For any $K^{\prime} \geq K$, we note $v^{K, K^{\prime}} \in L^{2}\left(\mathbb{T}^{2}\right)$ a solution of (3.11). Then, there exists $v^{K} \in$ $B V\left(\mathbb{T}^{2}\right) \cap L^{2}\left(\mathbb{T}^{2}\right)$ and a subsequence $\left(v^{K, K_{j}^{\prime}}\right)_{j \in \mathbb{N}}$ such that

$$
\lim _{j \rightarrow \infty}\left\|v^{K, K_{j}^{\prime}}-v^{K}\right\|_{1}=0
$$

and $\left(v^{K, K_{j}^{\prime}}\right)_{j \in \mathbb{N}}$ weakly converges in $L^{2}\left(\mathbb{T}^{2}\right)$ to $v^{K}$.

Moreover, for any such subsequence $\left(v^{K, K_{j}^{\prime}}\right)_{j \in \mathbb{N}}$, its limit $v^{K}$ is a solution of (3.10) and

$$
\lim _{j \rightarrow \infty} E_{K_{j}^{\prime}}\left(v^{K, K_{j}^{\prime}}\right)=\int_{\mathbb{T}^{2}}\left|\nabla v^{K}\right| .
$$

Proof. In order to simplify notations we will forget the index $K$ in $v^{K, K^{\prime}}$ and denote it by $v^{K^{\prime}}$, the prime symbol reminding us that the index $K^{\prime}$ refers to the minimization of $E_{K^{\prime}}$.

We remark first that, since for any $w \in \mathcal{W}_{s, u}^{K}, w$ is band-limited, we have

$$
\lim _{K^{\prime} \rightarrow \infty} E_{K^{\prime}}(w)=\int_{\mathbb{T}^{2}}|\nabla w| .
$$


Therefore, $\lim _{K^{\prime} \rightarrow \infty} E_{K^{\prime}}\left(u^{c}\right)$ exists (where $u^{c}$ is defined in (3.5)), and we know that there exists $C>0$ such that

$$
E_{K^{\prime}}\left(v^{K^{\prime}}\right) \leq E_{K^{\prime}}\left(u^{c}\right) \leq C .
$$

Unfortunately, we do not have any compactness theorem for $E_{K^{\prime}}$. However, we can boil down to compactness in $B V$ since for any $w \in L^{2}\left(\mathbb{T}^{2}\right)$, the frequency support of which is included in $\left\{-\frac{K}{2}+\frac{1}{N}, \ldots, \frac{K}{2}-\frac{1}{N}\right\}^{2}$,

$$
\begin{aligned}
\int_{\mathbb{T}^{2}}|w(x, y)| d x d y & =\int_{\mathbb{T}^{2}}\left|\frac{1}{N^{2}} \sum_{(k, l) \in \mathbb{Z}^{2}} \hat{w}\left(\frac{k}{N}, \frac{l}{N}\right) e^{2 i \pi\left(\frac{k x+l y}{N}\right)}\right| d x d y \\
& \leq \sum_{(k, l) \in \mathbb{Z}^{2}}\left|\hat{w}\left(\frac{k}{N}, \frac{l}{N}\right)\right| \\
& \leq(K N)^{2} \sup _{|k|,|l|<\frac{K N}{2}}\left|\hat{w}\left(\frac{k}{N}, \frac{l}{N}\right)\right| .
\end{aligned}
$$

Denote $\hat{w}_{k, l}$ the DFT (adapted to images of size $K^{\prime} N \times K^{\prime} N$ ) of $w$. We have

$$
\hat{w}_{k, l}=\sum_{m, n=0}^{K^{\prime} N-1} w\left(\frac{m}{K^{\prime}}, \frac{n}{K^{\prime}}\right) e^{-2 i \pi \frac{k m+l n}{K^{\prime} N}}=K^{\prime 2} \hat{w}\left(\frac{k}{N}, \frac{l}{N}\right),
$$

since $\hat{w}$ is supported by $\left\{-\frac{K}{2}+\frac{1}{N}, \ldots, \frac{K}{2}-\frac{1}{N}\right\}^{2}$ and $K^{\prime} \geq K$. Therefore, (3.14) yields

$$
\int_{\mathbb{T}^{2}}|w| \leq \frac{K^{2} N^{2}}{K^{\prime 2}} \sum_{m, n=0}^{K^{\prime} N-1}\left|w\left(\frac{m}{K^{\prime}}, \frac{n}{K^{\prime}}\right)\right| .
$$

Applying this to $\frac{\partial w}{\partial x}$ and $\frac{\partial w}{\partial y}$ for a $w \in \mathcal{W}_{s, u}^{K}$, we finally get

$$
\int_{\mathbb{T}^{2}}|\nabla w| \leq C^{\prime} E_{K^{\prime}}(w)
$$

where $C^{\prime}$ does not depend on $K^{\prime}$. This, combined with (3.13), yields

$$
\int_{\mathbb{T}^{2}}\left|\nabla v^{K^{\prime}}\right| \leq C^{\prime} C
$$

We can still do the same reasoning as that presented in the proof of Proposition 3.2 , which guarantees the existence of a subsequence $\left(v^{K_{j}^{\prime}}\right)_{j \in \mathbb{N}}$ and a function $v \in$ $B V\left(\mathbb{T}^{2}\right) \cap L^{2}\left(\mathbb{T}^{2}\right)$, such that $v^{K_{j}^{\prime}}$ converges in $L^{1}\left(\mathbb{T}^{2}\right)$ and converges weakly in $L^{2}\left(\mathbb{T}^{2}\right)$ to $v$.

We still have to prove that $v$ is a solution of the band-limited approximating restoration (3.10). First note that the weak convergence in $L^{2}$ ensures that $v \in \mathcal{W}_{s, u}^{K}$, since $\forall j, v^{K^{\prime}{ }_{j}}$ do belong to $\mathcal{W}_{s, u}^{K}$.

Let us now prove (3.12). We decompose

$$
E_{K_{j}^{\prime}}\left(v^{K^{\prime}{ }_{j}}\right)-\int_{\mathbb{T}^{2}}|\nabla v|=E_{K_{j}^{\prime}}\left(v^{K^{\prime}{ }_{j}}\right)-E_{K_{j}^{\prime}}(v)+E_{K_{j}^{\prime}}(v)-\int_{\mathbb{T}^{2}}|\nabla v| .
$$


Therefore, since $\lim _{K^{\prime} \rightarrow \infty} E_{K^{\prime}}(v)=\int_{\mathbb{T}^{2}}|\nabla v|$, it is then sufficient to prove that

$$
\lim _{j \rightarrow \infty} E_{K_{j}^{\prime}}\left(v^{K^{\prime}}{ }_{j}-v\right)=0
$$

to get (3.12).

Letting $w \in L^{2}\left(\mathbb{T}^{2}\right)$, the Fourier series of which is supported on $\left\{-\frac{K}{2}+\frac{1}{N}, \ldots\right.$, $\left.\frac{K}{2}-\frac{1}{N}\right\}^{2}$, we have

$$
E_{K^{\prime}}(w) \leq \frac{1}{K^{\prime 2}} \sum_{m, n=0}^{K^{\prime} N-1}\left|\frac{\partial w}{\partial x}\left(\frac{m}{K^{\prime}}, \frac{n}{K^{\prime}}\right)\right|+\frac{1}{K^{\prime 2}} \sum_{m, n=0}^{K^{\prime} N-1}\left|\frac{\partial w}{\partial y}\left(\frac{m}{K^{\prime}}, \frac{n}{K^{\prime}}\right)\right| .
$$

So, analyzing both summations separately, we have, since $K^{\prime} \geq K$,

$$
\frac{1}{K^{\prime 2}} \sum_{m, n=0}^{K^{\prime} N-1}\left|\frac{\partial w}{\partial x}\left(\frac{m}{K^{\prime}}, \frac{n}{K^{\prime}}\right)\right|=\frac{1}{K^{\prime 2}} \sum_{m, n=0}^{K^{\prime} N-1}\left|\frac{2 \pi}{\left(K^{\prime} N\right)^{2}} \sum_{k, l=-\frac{K^{\prime} N}{2}+1}^{\frac{K^{\prime} N}{2}} \frac{k}{K^{\prime} N} \hat{w}_{k, l} e^{2 i \pi \frac{k m+l n}{K^{\prime} N}}\right|
$$

Thus, since $\hat{w}_{k, l}=0$ for $|k|$ or $|l|$ strictly larger than $\frac{K N}{2}$, we have

$$
\begin{aligned}
\frac{1}{K^{\prime 2}} \sum_{m, n=0}^{K^{\prime} N-1}\left|\frac{\partial w}{\partial x}\left(\frac{m}{K^{\prime}}, \frac{n}{K^{\prime}}\right)\right| & \leq \frac{1}{K^{\prime 2}} \sum_{m, n=0}^{K^{\prime} N-1} \frac{\pi K^{2}}{K^{\prime 2}} \sup _{k, l}\left|\hat{w}_{k, l}\right| \\
& \leq \frac{\pi N^{2} K^{2}}{K^{\prime 2}} \sup _{k, l}\left|\hat{w}_{k, l}\right| .
\end{aligned}
$$

Once again, we just remark that, since $K^{\prime} \geq K$, we have $\hat{w}_{k, l}=K^{\prime 2} \hat{w}\left(\frac{k}{N}, \frac{l}{N}\right)$ and we obtain

$$
\frac{1}{K^{\prime 2}} \sum_{m, n=0}^{K^{\prime} N-1}\left|\frac{\partial w}{\partial x}\left(\frac{m}{K^{\prime}}, \frac{n}{K^{\prime}}\right)\right| \leq \pi N^{2} K^{2} \int_{\mathbb{T}^{2}}|w| .
$$

So, there exists $C>0$, which does not depend on $K^{\prime}$, such that for any $w \in L^{2}\left(\mathbb{T}^{2}\right)$, the Fourier series of which is supported by $\left\{-\frac{K}{2}+\frac{1}{N}, \ldots, \frac{K}{2}-\frac{1}{N}\right\}^{2}$, we have

$$
E_{K_{j}^{\prime}}(w) \leq C \int_{\mathbb{T}^{2}}|w|
$$

Applying this to $\left(v^{K^{\prime}{ }_{j}}-v\right)$ yields $\lim _{j \rightarrow \infty} E_{K_{j}^{\prime}}\left(v^{K^{\prime}{ }_{j}}-v\right)=0$.

Therefore, (3.12) holds and for any $w \in \mathcal{W}_{s, u}^{K}$, we have

$$
\int_{\mathbb{T}^{2}}|\nabla w|=\lim _{j \rightarrow \infty} E_{K_{j}^{\prime}}(w) \geq \lim _{j \rightarrow \infty} E_{K_{j}^{\prime}}\left(v^{K_{j}^{\prime}}\right)=\int_{\mathbb{T}^{2}}|\nabla v|,
$$

which achieves the proof.

The following proposition shows that $\left(v^{K}\right)_{K \in \mathbb{N}}$ allows us to approximate a solution $v$ of (3.6).

Proposition 3.8. Let $N$ be an integer, $u \in l^{\infty}\left(\mathbb{Z}^{2}\right) N$-periodic, and $s \in L^{2}\left(\mathbb{T}^{2}\right)$ satisfying the hypotheses of the Proposition 3.2. If for any $K>1$, we note $v^{K} \in$ 
$L^{2}\left(\mathbb{T}^{2}\right)$ a solution of (3.10), we know that there exists $v \in \mathcal{W}_{s, u}$ and a subsequence $\left(v^{K_{j}}\right)_{j \in \mathbb{N}}$ such that

$$
\lim _{j \rightarrow \infty}\left\|v^{K_{j}}-v\right\|_{1}=0
$$

and $\left(v^{K_{j}}\right)_{j \in \mathbb{N}}$ weakly converges in $L^{2}$ to $v$.

Moreover, for any such subsequence $\left(v^{K_{j}}\right)_{j \in \mathbb{N}}$, its limit $v$ is a solution of (3.6) and

$$
\lim _{j \rightarrow \infty} \int_{\mathbb{T}^{2}}\left|\nabla v^{K_{j}}\right|=|D v|\left(\mathbb{T}^{2}\right)
$$

Proof. First note that the sequence $\left(\left|D v^{K}\right|\left(\mathbb{T}^{2}\right)\right)_{K \in \mathbb{N}}$ decreases. So, the same reasoning as that presented in the proof of the Proposition 3.2 yields that there exists $v \in B V(\mathbb{T}) \cap L^{2}\left(\mathbb{T}^{2}\right)$ and a subsequence $\left(v^{K_{j}}\right)_{j \in \mathbb{N}}$ such that $v_{K_{j}}$ converges in $L^{1}$ and weakly converges in $L^{2}$ to $v$. Moreover, we have

$$
|D v|\left(\mathbb{T}^{2}\right) \leq \liminf _{j \rightarrow \infty}\left|D v^{K_{j}}\right|\left(\mathbb{T}^{2}\right) .
$$

Once again, the weak convergence in $L^{2}$ ensures that $v \in \mathcal{W}_{s, u}$.

Nevertheless, we still have to prove that $|D v|\left(\mathbb{T}^{2}\right)$ is minimum among the functions of $\mathcal{W}_{s, u}$. For that, we are going to build a sequence of functions, indexed by $K$, of elements of $\mathcal{W}_{s, u}^{K}$ and which minimizes the total variation.

Letting $K>1$ be an integer, we define for any $(k, l) \in \mathbb{Z}^{2}$

$$
\hat{h}_{K}\left(\frac{k}{N}, \frac{l}{N}\right)= \begin{cases}\left(1-\frac{|2 k|}{K N}\right)\left(1-\frac{|2 l|}{K N}\right) & \text { for }|k| \text { and }|l| \leq \frac{K N}{2} \\ 0 & \text { otherwise. }\end{cases}
$$

For any $K>1, h_{K} \in L^{2}\left(\mathbb{T}^{2}\right) \cap L^{1}\left(\mathbb{T}^{2}\right)$ and $\int_{\mathbb{T}^{2}}\left|h_{K}\right|=1$; indeed $\int_{\mathbb{T}^{2}} h_{K}=1$ since $\hat{h}_{K}(0,0)=1$ and $h_{K} \geq 0$ since it is the convolution of two positive functions (which are obtained by convolving the Fourier series of a function with itself).

We define an operator $P_{K}$ from $\mathcal{W}_{s, u}$ onto $\mathcal{W}_{s, u}^{K}$ by

$$
P_{K}(w)=h_{K} * w+w_{K}^{\prime},
$$

where $w \in \mathcal{W}_{s, u}$ and $w_{K}^{\prime}$ is defined as follows: $\widehat{w_{K}^{\prime}}$ is supported on $\left\{-\frac{1}{2}+\frac{1}{N}, \ldots, \frac{1}{2}\right\}^{2}$ and for any $(\xi, \eta) \in\left\{-\frac{N}{2}-1, \ldots, \frac{N}{2}\right\}^{2}$,

$\widehat{w_{K}^{\prime}}\left(\frac{\xi}{N}, \frac{\eta}{N}\right)$

$=\frac{1}{\hat{s}\left(\frac{\xi}{N}, \frac{\eta}{N}\right)} \sum_{\left(k^{\prime}, l^{\prime}\right) \in \mathbb{Z}^{2}} \hat{s}\left(\frac{\xi}{N}+k^{\prime}, \frac{\eta}{N}+l^{\prime}\right) \hat{w}\left(\frac{\xi}{N}+k^{\prime}, \frac{\eta}{N}+l^{\prime}\right)\left[1-\hat{h}_{K}\left(\frac{\xi}{N}+k^{\prime}, \frac{\eta}{N}+l^{\prime}\right)\right]$.

It is easy to check using (3.4) and (3.9) that $P_{K}(w) \in \mathcal{W}_{s, u}^{K}$ for any $w \in \mathcal{W}_{s, u}$.

Let us show that $P_{K}(w)$ converges to $w$ in $L^{2}\left(\mathbb{R}^{2}\right)$ when $K$ tends to infinity. Letting $K>3$, we have

$$
\left\|k_{K} * w-w\right\|_{2}^{2}=\frac{1}{N^{2}} \sum_{(k, l) \in \mathbb{Z}^{2}}\left|\hat{w}\left(\frac{k}{N}, \frac{l}{N}\right)\right|^{2}\left|1-\hat{h}_{K}\left(\frac{k}{N}, \frac{l}{N}\right)\right|^{2} .
$$


Now let us denote $C_{K}=K N\left(1-\left(1-\frac{1}{\log K}\right)^{\frac{1}{2}}\right)$ and split the sum into two pieces: $S_{1}$, the sum over all the indexes whose magnitudes are below $C_{K}$; and $S_{2}$, the sum over the reminding indexes.

Due to the particular form of $\hat{h}$, we have for indexes $k$ and $l$ that concern the sum $S_{1},\left|1-\hat{h}_{K}\left(\frac{k}{N}, \frac{l}{N}\right)\right| \leq \frac{1}{\log K}$. This yields

$$
S_{1} \leq \frac{1}{(\log K)^{2}}\|w\|_{2}^{2},
$$

which guarantees that $\lim _{K \rightarrow \infty} S_{1}=0$. Concerning $S_{2}$, we get

$$
S_{2} \leq \sum_{|k| \text { or }|l|>C_{K}}\left|\hat{w}\left(\frac{k}{N}, \frac{k}{N}\right)\right|^{2},
$$

since $\left|1-\hat{h}_{K}\left(\frac{k}{N}, \frac{l}{N}\right)\right| \leq 1$. Therefore, $\lim _{K \rightarrow \infty} S_{2}=0$ and we get

$$
\lim _{K \rightarrow \infty}\left\|k_{K} * w-w\right\|_{2}=0 .
$$

The same kind of calculus yields

$$
\lim _{K \rightarrow \infty}\left\|w_{K}^{\prime}\right\|_{2}=0
$$

and we finally have

$$
\lim _{K \rightarrow \infty}\left\|P_{K}(w)-w\right\|_{2}=0 .
$$

Now we are going to show that $\lim _{K \rightarrow \infty}\left|D P_{K}(w)\right|\left(\mathbb{T}^{2}\right)$ exists and is smaller than $|D w|\left(\mathbb{T}^{2}\right)$. This is basically a consequence of the fact that $\left|D\left(h_{K} * w\right)\right|\left(\mathbb{T}^{2}\right) \leq$ $|D(w)|\left(\mathbb{T}^{2}\right)$ and that $\left|D w_{K}^{\prime}\right|\left(\mathbb{T}^{2}\right)$ tends to 0 when $K$ tends to infinity. Let us detail this.

Let $w \in \mathcal{W}_{s, u}$ and $\varphi \in C^{1}\left(\mathbb{T}^{2}, \mathbb{R}^{2}\right)$ be compactly supported such that $\|\varphi\|_{\infty} \leq 1$. We have

$$
\begin{aligned}
\int_{\mathbb{T}^{2}} P_{K}(w) \operatorname{div}(\varphi) & =\int_{\mathbb{T}^{2}} w \operatorname{div}\left(h_{K} * \varphi\right)+\int_{\mathbb{T}^{2}} w_{K}^{\prime} \operatorname{div}(\varphi), \\
& \leq|D w|\left(\mathbb{T}^{2}\right)+\int_{\mathbb{T}^{2}} w_{K}^{\prime} \operatorname{div}(\varphi) .
\end{aligned}
$$

So, since $w_{K}^{\prime}$ and $\operatorname{div}(\varphi)$ belong to $L^{2}\left(\mathbb{T}^{2}\right)$, we can compute $I_{1}=\int_{\mathbb{T}^{2}} w_{K}^{\prime} \operatorname{div}(\varphi)$ in frequency domain and this yields the upper bound (we note $\varphi=\left(\varphi_{1}, \varphi_{2}\right), \overline{\hat{\varphi}_{i}}$ being the complex conjugate of $\hat{\varphi}_{i}$ for $i=1,2$ )

$$
\begin{aligned}
\left|I_{1}\right| & =\frac{2 \pi}{N^{2}}\left|\sum_{-\frac{N}{2}+1 \leq \xi, \eta \leq \frac{N}{2}} \widehat{w_{K}^{\prime}}\left(\frac{\xi}{N}, \frac{\eta}{N}\right)\left[\overline{\frac{\xi}{N}} \overline{\hat{\varphi}_{1}\left(\frac{\xi}{N}, \frac{\eta}{N}\right)}+\overline{\frac{\eta}{N}} \widehat{\hat{\varphi}_{2}\left(\frac{\xi}{N}, \frac{\eta}{N}\right)}\right]\right| \\
& \leq 2 \pi\left\|w_{K}^{\prime}\right\|_{2}\left(\sum_{-\frac{N}{2}+1 \leq \xi, \eta \leq \frac{N}{2}}\left|\frac{\xi}{N} \hat{\varphi}_{1}\left(\frac{\xi}{N}, \frac{\eta}{N}\right)+\frac{\eta}{N} \hat{\varphi}_{2}\left(\frac{\xi}{N}, \frac{\eta}{N}\right)\right|^{2}\right)^{\frac{1}{2}} \\
& \leq 2 \pi\left\|w_{K}^{\prime}\right\|_{2}\left(\left\|\varphi_{1}\right\|_{2}^{2}+\left\|\varphi_{2}\right\|_{2}^{2}\right)^{\frac{1}{2}} \\
& \leq 2 \sqrt{2} \pi N\left\|w_{K}^{\prime}\right\|_{2} .
\end{aligned}
$$


Therefore, for any $\varepsilon>0$ and any $w \in \mathcal{W}_{s, u}$ there exists $K_{\varepsilon}>1$ such that for $K \geq K_{\varepsilon}$

$$
\left|D P_{K}(w)\right|\left(\mathbb{T}^{2}\right) \leq|D w|\left(\mathbb{T}^{2}\right)+\epsilon .
$$

So, since $|D w|\left(\mathbb{T}^{2}\right) \leq \liminf _{K \rightarrow \infty}\left|D P_{K}(w)\right|$, we have for any $w \in \mathcal{W}_{s, u}$

$$
\lim _{K \rightarrow \infty}\left|D P_{K}(w)\right|\left(\mathbb{T}^{2}\right)=|D w|\left(\mathbb{T}^{2}\right) .
$$

So, in particular, for a solution $w$ of (3.6), we have

$$
|D w|\left(\mathbb{T}^{2}\right) \leq\left|D v^{K_{j}}\right|\left(\mathbb{T}^{2}\right) \leq\left|D P_{K_{j}}(w)\right|\left(\mathbb{T}^{2}\right),
$$

and then $|D w|\left(\mathbb{T}^{2}\right)=\lim _{j \rightarrow \infty}\left|D v^{K_{j}}\right|\left(\mathbb{T}^{2}\right)$.

This, at last, guarantees

$$
|D v|\left(\mathbb{T}^{2}\right) \leq \lim _{j \rightarrow \infty}\left|D v^{K_{j}}\right|\left(\mathbb{T}^{2}\right)=|D w|\left(\mathbb{T}^{2}\right) \leq|D v|\left(\mathbb{T}^{2}\right),
$$

which finishes the proof.

We remark first that we could have stated, in the case of the band-limited approximating restoration (3.10), some propositions similar to Proposition 3.3 (the "weak uniqueness") and Proposition 3.6 (that deals with the preservation of cylindric images for (3.6)). So, since any function $w \in \mathcal{W}_{s, u}^{K}$ is continuously differentiable and since for any such function $\left\{(x, y) \in \mathbb{T}^{2}, \nabla w=0\right\}$ is of null measure, we can expect any solution $v^{K}$ of (3.10) to be cylindric, given an initial cylindric data. Therefore, a solution $v$ of (3.6) obtained as a limit of a subsequence $\left(v^{K_{j}}\right)_{j \in \mathbb{N}}$ is also cylindric.

Now, as we said in the introduction of this section, we can only compute a solution of the solvable restoration (given by (3.11)). Now we are going to show the consequence of the change in the minimizing energy on the behavior of the restoration when dealing with cylindric images.

Proposition 3.9. Let $N, K$, and $K^{\prime}$ be some integers with $K^{\prime} \geq K>1$, $(\alpha, \beta) \in \mathbb{Z}^{2} \backslash\{(0,0)\}$, and $u \in l^{\infty}\left(\mathbb{Z}^{2}\right) N$-periodic and cylindric along the direction $(\alpha, \beta)$. For any kernel $s=h * s^{e}$ with $h \in L^{2}\left(\mathbb{T}^{2}\right)$ such that $\hat{h}$ does not vanish on $\left\{-\frac{1}{2}+\frac{1}{N}, \ldots, \frac{1}{2}\right\}^{2}$, the solvable restoration (3.11) admits a solution whose Fourier series is supported on

$$
S_{\alpha^{\prime}, \beta^{\prime}}=\left\{(k, l) \in\left\{-\frac{K}{2}+\frac{1}{N}, \ldots, \frac{K}{2}-\frac{1}{N}\right\}^{2}, \beta^{\prime} k-\alpha^{\prime} l \in K^{\prime} \mathbb{Z}\right\},
$$

where $\left(\alpha^{\prime}, \beta^{\prime}\right) \in \mathbb{Z}^{2}$ are such that $\alpha^{\prime} \wedge \beta^{\prime}=1$ ( $\wedge$ denotes the largest common divisor) and $\left(\alpha^{\prime}, \beta^{\prime}\right)$ defines the same direction as $(\alpha, \beta)$.

Remark that, modulo the uniqueness (we could state a proposition similar to Proposition 3.3 in the case of (3.11)), this proposition means that the results of the solvable restoration are not fully cylindric (see the thick line on Figure 2).

Moreover, for $(\alpha, \beta) \in \mathbb{Z}^{2} \backslash\{(0,0)\}$, we can define $\left(\alpha^{\prime}, \beta^{\prime}\right) \in \mathbb{Z}^{2}$ as a couple representing the same direction as $(\alpha, \beta)$, but such that $\alpha^{\prime} \wedge \beta^{\prime}=1$. Then, since $S_{\alpha^{\prime}, \beta^{\prime}}$ is included in $S_{\alpha, \beta}$, the use of such a couple $\left(\alpha^{\prime}, \beta^{\prime}\right)$ in the statement of Proposition 3.9 yields a stronger result.

Proof. The proof is similar to the one of Proposition 3.6, except that we have to take care of the fact that $E_{K^{\prime}}(w)$ takes into account only the values of $w$ on a discrete set. 


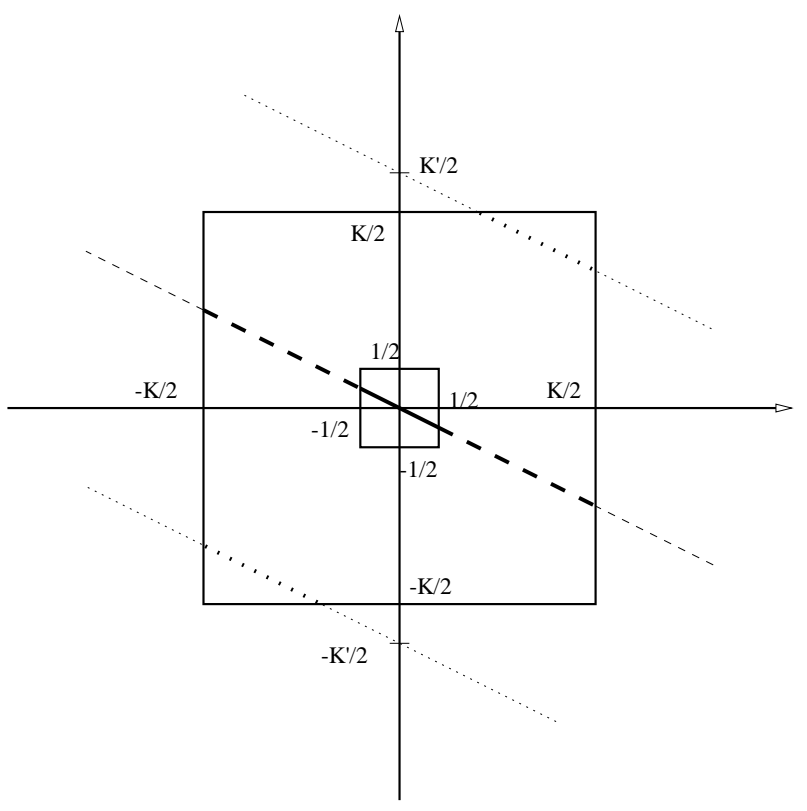

FIG. 2. The solid thick line represents the initial spectrum support (cylindric along the direction $(2,-1))$. The dashed lines (thick and thin) represent the restriction to the direction $(2,-1)$ of the possible spectrum support after minimizing $E_{K^{\prime}}$. The dotted lines (thick and thin) represent the restriction to wrong directions of the possible spectrum support after minimizing $E_{K^{\prime}}$. The thick lines (solid, dashed, and dotted) represent the possible spectrum support after minimizing $E_{K^{\prime}}$ among $\mathcal{W}_{s, u}^{K}$.

Let $\left(\alpha^{\prime}, \beta^{\prime}\right) \in \mathbb{Z}^{2}$ be such as in the proposition.

Letting $K^{\prime} \geq K, v \in \mathcal{W}_{s, u}^{K}$, be a solution of (3.11) and $M \in \mathbb{N}$, we define the function $v_{M} \in L^{2}\left(\mathbb{T}^{2}\right)$, in frequency domain, by

$$
\widehat{v_{M}}\left(\frac{k}{N}, \frac{l}{N}\right)=\hat{v}\left(\frac{k}{N}, \frac{l}{N}\right) \frac{1}{2 M+1} \sum_{t=-M}^{M} e^{-2 i \pi \frac{\left(k \beta^{\prime}-l \alpha^{\prime}\right)}{K^{\prime} N} t}
$$

for any $(k, l) \in \mathbb{Z}^{2}$.

There is an analogy between this $v_{M}$ and the one defined by (3.8) in that for any $(m, n) \in\left\{0, \ldots, K^{\prime} N-1\right\}^{2}$

$$
v_{M}\left(\frac{m}{K^{\prime}}, \frac{n}{K^{\prime}}\right)=\frac{1}{2 M+1} \sum_{t=-M}^{M} v\left(\frac{m}{K^{\prime}}-\beta^{\prime} t, \frac{n}{K^{\prime}}+\alpha^{\prime} t\right) .
$$

Indeed, since $v \in \mathcal{W}_{s, u}^{K}$ and $K^{\prime} \geq K$, we have

$$
\begin{aligned}
\left(\widehat{v_{M}}\right)_{k, l} & =K^{\prime 2} \widehat{v_{M}}\left(\frac{k}{N}, \frac{l}{N}\right) \\
& =K^{\prime 2} \hat{v}\left(\frac{k}{N}, \frac{l}{N}\right) \frac{1}{2 M+1} \sum_{t=-M}^{M} e^{-2 i \pi \frac{\left(k \beta^{\prime}-l \alpha^{\prime}\right)}{K^{\prime} N} t} \\
& =\hat{v}_{k, l} \frac{1}{2 M+1} \sum_{t=-M}^{M} e^{-2 i \pi \frac{\left(k \beta^{\prime}-l \alpha^{\prime}\right)}{K^{\prime} N} t}
\end{aligned}
$$

which in space domain yields (3.17). 
Similarly, we also have for any $(m, n) \in\left\{0, \ldots, K^{\prime} N-1\right\}^{2}$

$$
\nabla v_{M}\left(\frac{m}{K^{\prime}}, \frac{n}{K^{\prime}}\right)=\frac{1}{2 M+1} \sum_{t=-M}^{M} \nabla v\left(\frac{m}{K^{\prime}}-\beta^{\prime} t, \frac{n}{K^{\prime}}+\alpha^{\prime} t\right)
$$

and so

$$
E_{K^{\prime}}\left(v_{M}\right) \leq E_{K^{\prime}}(v) .
$$

(Note that it is the point where the $v_{M}$ defined by (3.8) fail.)

We remark that $\left\{w \in L^{2}\left(\mathbb{T}^{2}\right), \operatorname{Supp}(\hat{w}) \subset\left\{-\frac{K}{2}+\frac{1}{N}, \ldots, \frac{K}{2}-\frac{1}{N}\right\}\right\}$ is a finite dimensional space and that $E_{K^{\prime}}(w)=0$ implies $\int_{\mathbb{T}^{2}}|\nabla w|=0$. So there exists $C>0$ such that for any $w \in L^{2}\left(\mathbb{T}^{2}\right)$ satisfying $\operatorname{Supp}(\hat{w}) \subset\left\{-\frac{K}{2}+\frac{1}{N}, \ldots, \frac{K}{2}-\frac{1}{N}\right\}$

$$
\int_{\mathbb{T}^{2}}|\nabla w| \leq C E_{K^{\prime}}(w) .
$$

So, $\int_{\mathbb{T}^{2}}\left|\nabla v_{M}\right|$ is bounded and, similar to Proposition 3.6, we know that there exists $v_{M_{j}}$ that converges in $L^{1}\left(\mathbb{T}^{2}\right)$ to a function $v^{\prime} \in B V\left(\mathbb{T}^{2}\right) \cap L^{2}\left(\mathbb{T}^{2}\right)$.

Moreover, as shown before, there exists $C^{\prime}>0$ such that for any $w \in L^{2}\left(\mathbb{T}^{2}\right)$ such that $\operatorname{Supp}(\hat{w}) \subset\left\{-\frac{K}{2}+\frac{1}{N}, \ldots, \frac{K}{2}-\frac{1}{N}\right\}$

$$
E_{K^{\prime}}(w) \leq C^{\prime} \int_{\mathbb{T}^{2}}|w|
$$

So, $\lim _{j \rightarrow \infty} E_{K^{\prime}}\left(v_{M_{j}}-v^{\prime}\right)=0$ and

$$
E_{K^{\prime}}\left(v^{\prime}\right)=\lim _{j \rightarrow \infty} E_{K^{\prime}}\left(v_{M_{j}}\right) \leq E_{K^{\prime}}(v) .
$$

Moreover, since $v_{M_{j}}$ converges to $v^{\prime}$ in $L^{1}\left(\mathbb{T}^{2}\right)$, looking at (3.16), we finally have for any $(k, l) \in \mathbb{Z}^{2}$

$$
\widehat{v^{\prime}}\left(\frac{k}{N}, \frac{l}{N}\right)= \begin{cases}\hat{v}\left(\frac{k}{N}, \frac{l}{N}\right) & \text { if }\left(k \beta^{\prime}-l \alpha^{\prime}\right) \in K^{\prime} N \mathbb{Z}, \\ 0 & \text { otherwise. }\end{cases}
$$

This ensures that $v^{\prime} \in \mathcal{W}_{s, u}^{K}$ and finishes the proof of the announced result.

We remark, however, that the solution $v^{\prime}$ of the solvable restoration (3.11) given in the proof of the proposition satisfies

$$
v^{\prime}\left(\frac{m}{K^{\prime}}, \frac{n}{K^{\prime}}\right)=v^{\prime}\left(\frac{m}{K^{\prime}}-\beta^{\prime} t, \frac{n}{K^{\prime}}+\alpha^{\prime} t\right)
$$

for any $(m, n) \in\left\{0, \ldots, K^{\prime} N-1\right\}^{2}$ and any $t \in \mathbb{Z}$.

We remark once again that we could have stated a proposition similar to Proposition 3.6 (which ensures the preservation of cylindric function for (3.6)) in the case of the band-limited approximating restoration (3.10). So, this proposition has to be regarded under the scope of Proposition 3.7 (a consistency proposition) which guarantees that for $K^{\prime}$ large the cylindric sight of the result is preponderant. Moreover, we have the following corollary.

Corollary 3.10. Let $N$ and $K$ be two integers and $(\alpha, \beta) \in \mathbb{Z}^{2} \backslash\{(0,0)\}$. There exists $K^{\prime} \geq K$ beyond which the solutions of (3.11) are cylindric along the direction $(\alpha, \beta)$, for any kernel $s=h * s^{e}$ (see (3.2)), with $h \in L^{2}\left(\mathbb{T}^{2}\right)$ such that $\hat{h}$ does not 
vanish on $\left\{-\frac{1}{2}+\frac{1}{N}, \ldots, \frac{1}{2}\right\}^{2}$ and any $u \in l^{\infty}\left(\mathbb{Z}^{2}\right) N$-periodic and cylindric along the direction $(\alpha, \beta)$.

Indeed, looking at the definition of $S_{\alpha^{\prime}, \beta^{\prime}}$ (see Proposition 3.9), we see that for $K^{\prime}$ large the lines $\beta^{\prime} k-\alpha^{\prime} l= \pm K^{\prime}$ do not intersect the square $\left\{-\frac{K}{2}+\frac{1}{N}, \ldots, \frac{K}{2}-\frac{1}{N}\right\}^{2}$ (see Figure 2). Therefore, for $K^{\prime}$ large, $S_{\alpha^{\prime}, \beta^{\prime}}$ is reduced to the trail of the line $\beta^{\prime} k-\alpha^{\prime} l=0$ on $\left\{-\frac{K}{2}+\frac{1}{N}, \ldots, \frac{K}{2}-\frac{1}{N}\right\}^{2}$ and the solutions of (3.11) are cylindric along the direction $(\alpha, \beta)$.

Note also that the case $K^{\prime}=K$ is sufficient to preserve the functions cylindric along the axis directions (the cases where $(\alpha, \beta)=(1,0)$ or $(\alpha, \beta)=(0,1))$.

3.3. Some other variational restoration. As we said previously, most of the results presented above are also true for restorations based on minimization of other kind of energies. In this section, we are first going to study a particular case which yields a linear reconstruction, and we will then explain the reasons that let us think the total variation is the most adapted to image restoration.

3.3.1. Linear reconstructions associated with a variational restoration. There is an important link between linear reconstructions and some variational minimization of the form

$$
Z_{L}(u)=\operatorname{argmin}\left(\int_{\mathbb{T}^{2}}|L(w)|^{2}\right), \text { among } w \in \mathcal{W}_{s, u},
$$

where $L$ is a linear and translation invariant operator. (Note that variational minimizations of the same kind have already been introduced in [12].)

In order to describe this link, let us focus on the particular case where $L=\nabla$. This leads us to search solutions of

$$
Z(u)=\operatorname{argmin}\left(\int_{\mathbb{T}^{2}}|\nabla w|^{2}\right), \text { among } w \in \mathcal{W}_{s, u} .
$$

Let us state the following proposition which gives the explicit form of a solution of (3.19). Note that this proposition could be stated in the case of $Z_{L}$ for most of the linear and translation invariant operator $L$. (We will explain this more precisely after having stated the proposition.)

Proposition 3.11. Let $N \in \mathbb{N}, u \in l^{\infty}\left(\mathbb{Z}^{2}\right) N$-periodic, and let $s \in L^{2}\left(\mathbb{T}^{2}\right)$ such that $\hat{s}$ does not vanish on $\left\{-\frac{1}{2}+\frac{1}{N}, \ldots, \frac{1}{2}\right\}^{2}$; then (3.19) defines a linear, translation invariant operator continuous from $\left\{u \in l^{\infty}\left(\mathbb{Z}^{2}\right)\right.$, $u$ is $N$-periodic $\}$ to $L^{2}\left(\mathbb{T}^{2}\right)$. Moreover, (3.19) admits a unique solution $v$ given by

for any $(\xi, \eta) \in\left\{-\frac{N}{2}-1, \ldots, \frac{N}{2}\right\}^{2} \backslash\{(0,0)\}$ and any $(k, l) \in \mathbb{Z}^{2}$,

$$
\hat{v}\left(\frac{\xi}{N}+k, \frac{\eta}{N}+l\right)=\frac{\overline{\hat{s}}\left(\frac{\xi}{N}+k, \frac{\eta}{N}+l\right)}{\left[\left(\frac{\xi}{N}+k\right)^{2}+\left(\frac{\eta}{N}+l\right)^{2}\right] \psi\left(\frac{\xi}{N}, \frac{\eta}{N}\right)} \hat{u}_{\frac{\xi}{N}, \frac{\eta}{N}},
$$

where $\overline{\hat{s}}$ denotes the complex conjugate of $\hat{s}$ and

$$
\psi\left(\frac{\xi}{N}, \frac{\eta}{N}\right)=\sum_{(k, l) \in \mathbb{Z}^{2}} \frac{\left|\hat{s}\left(\frac{\xi}{N}+k, \frac{\eta}{N}+l\right)\right|^{2}}{\left(\frac{\xi}{N}+k\right)^{2}+\left(\frac{\eta}{N}+l\right)^{2}},
$$

and for any $(k, l) \in \mathbb{Z}^{2}$

$$
\hat{v}(k, l)= \begin{cases}\hat{u}_{0,0} & \text { for }(k, l)=(0,0), \\ \hat{s}_{0,0} & \text { otherwise. }\end{cases}
$$

A proof of this proposition can found in [15]. 
As we said previously, this proposition can be generalized to find solutions of problems of the form (3.18). Indeed, if we note $L(w)=h * w$ and if there exists $C>0$ such that $\left|\hat{h}\left(\frac{k}{N}, \frac{l}{N}\right)\right|^{2} \geq C$ for $(k, l) \neq(0,0)$, we simply have to replace, in Proposition $3.11,\left(\frac{\xi}{N}+k\right)^{2}+\left(\frac{\eta}{N}+l\right)^{2}$ by $\left|\hat{h}\left(\frac{\xi}{N}+k, \frac{\eta}{N}+l\right)\right|^{2}$. Note that this holds for any differential operator.

Note also that, when $\hat{s} \in l^{1}$, the interpolation defined by (3.19) can be extended to $l^{\infty}\left(\mathbb{Z}^{2}\right)$ (by interpolating the Fourier series of the convolution kernel) in such a way that it satisfies the hypotheses of the Propositions 2.7 and 2.9. Therefore, since we can see in Proposition 3.11 that it puts 0 at lost frequencies (where $\hat{s}=0$ ), Propositions 2.7 and 2.9 yield results similar to Propositions 3.5 and 3.6 but adapted to the problem (3.19).

We remark also that for any linear and translation invariant interpolation operator (defined by an $h$ such that $|\nabla h| \in L^{2}\left(\mathbb{T}^{2}\right)$ and $\hat{h}\left(\frac{\xi}{N}, \frac{\eta}{N}\right) \neq 0$ for any $(\xi, \eta) \in\{-N / 2+$ $\left.1, \ldots, N / 2\}^{2}\right),(3.19)$ applied with the kernel

$$
\hat{s}\left(\frac{\xi}{N}+k, \frac{\eta}{N}+l\right)=\frac{\left(\left(\frac{\xi}{N}+k\right)^{2}+\left(\frac{\eta}{N}+l\right)^{2}\right) \quad \overline{\hat{h}}\left(\frac{\xi}{N}+k, \frac{\eta}{N}+l\right)}{\sum_{\left(k^{\prime}, l^{\prime}\right) \in \mathbb{Z}^{2}}\left[\left(\frac{\xi}{N}+k^{\prime}\right)^{2}+\left(\frac{\eta}{N}+l^{\prime}\right)^{2}\right]\left|\hat{h}\left(\frac{\xi}{N}+k^{\prime}, \frac{\eta}{N}+l^{\prime}\right)\right|^{2}}
$$

defines the same interpolation as the one simply defined by $h$.

Therefore, this analysis gives a parallel between linear, translation invariant reconstructions and solutions of (3.19). This parallel can be used to describe linear reconstructions but makes useless the use of (3.19) to reconstruct images.

3.3.2. Discussion on the energy choice. Now we are going to argue for the choice of the total variation in image restoration within the family of energies

$$
E_{L, r}(w)=\int_{\mathbb{T}^{2}}|L(w)|^{r},
$$

where $r \geq 1$ and $L$ is a linear translation invariant isotropic operator; we moreover consider it homogeneous (typically a differential operator of a fixed order). Homogeneous is used in the sense that

$$
\begin{array}{r}
\exists \operatorname{ord}(L) \in \mathbb{R}, \forall C>0, \forall w \text { such that } L(w) \text { exists everywhere, } \\
L(w(C .))(x, y)=C^{\text {ord }(L)} L(w)(C x, C y)
\end{array}
$$

For commodity we do not distinguish yet the total variation of a function $w$ from $\int_{\mathbb{T}^{2}}|\nabla w|$.

First note that within this family the case $r=1$ is the most interesting since for $r>1$ the energy $E_{L, r}(w)$ does penalize more a point at which $|L(w)|$ is large than it penalizes several points having smaller $|L(w)|$. This is simply due to the fact that $f(t)=t^{r}$ is strictly convex for $r>1$ and can be regarded as a consequence of the homogeneity of $E_{L, r}(w)$ with regard to multiplications of $w$ by constant. We do believe such a behavior is harmful to image quality since it will split large $|L(w)|$ into several pieces without regard to their orientation. (This orientation generally oscillates due to the data fidelity term.) This does generally create oscillations close to points where $|L(w)|$ is large (generally edges). This behavior can be observed in the case of $E_{\nabla, 2}$.

Let us focus on the case where $r=1$. If we consider an image cylindric along a direction $(\alpha, \beta)$ (which models an edge) and a dilation (or contraction) along the 
direction $(\alpha, \beta)$ (this modifies the edge smoothness), in such a case the homogeneity of $E_{L, 1}(w)$ with regard to this dilation is $\operatorname{ord}(L)-1$. So, if $\operatorname{ord}(L)$ is strictly larger than $1, E_{L, 1}(w)$ will penalize more sharp edges than smooth ones (indeed, $C^{\text {ord }}(L)-1$ tends to infinity with $C$ ) and similarly to previously, the minimization of $E_{L, 1}(w)$ tends to create oscillations close to edges. Moreover, natural images generally present sharp edges, so we cannot afford to forbid them during the restoration. (Note that this is an intuitive way to talk about the Sobolev embedding theorem which in 1D shows that the case $\operatorname{ord}(L)=1$ and $r=1$ is the limit one beyond which the functions are smoother than continuous.)

A simple way to sum up this is the following $1 \mathrm{D}$ proposition.

Proposition 3.12. For any signal $w \in B V(\mathbb{T})$ and any homeomorphism $f$ from $\mathbb{T}$ to $\mathbb{T}$,

$$
|D(w \circ f)|(\mathbb{T})=|D w|(\mathbb{T}) .
$$

This result has already been introduced in [8].

Now, the total variation extends $\int_{\mathbb{T}^{2}}|\nabla w|$ in such a way that it is lower semicontinuous and satisfies some compactness properties. Therefore, the total variation permits us to state proper results.

There exist, of course, other kinds of energies. Let us cite entropy which has already been used to restore images (see [27]). The entropy is generally defined, for a positive function $w$, by

$$
E(w)=-\int_{\mathbb{T}^{2}} w(x, y) \log \left(\frac{w(x, y)}{\int_{\mathbb{T}^{2}} w}\right) d x d y .
$$

We remark that a small change of $f$ in a dark part of the image a priori induces a larger change in the value of the entropy than the same change in a bright region. Therefore, we believe such a restoration is not adapted to image restoration since it may not behave in the same way in dark and bright regions of the image.

4. Experiments. We illustrate in this section some propositions and the methods described above. The methods are the duplication interpolation, the bicubic reconstruction (see [20]), the "zero-padding" interpolation, a reconstruction using a Prolate function, and the total variation based restoration. (These are respectively presented on figures from up to down and from left to right.) In all the experiments, the data is deduced from a reference image by keeping the low frequencies corresponding to a subsampling of a factor 4 . The reference image is also displayed on the lower right-hand corner of the figures. In order to compare the reconstructed image in front of the reference, all the presented experiments are zooms of level 4. (The initial image numbers of row and of column are multiplied by 4.) Moreover, they are generally displayed both in space and frequency domain. In this latter case, the presented images are the spectrum modulus raised to the power 0.01. The experiments are presented as follows. We start from reconstruction of cylindric image and leave this framework to conclude with natural images. At last, we give some statistics which give the $l^{2}$-norm of the difference between the reconstructions and the reference image and illustrate the importance of the hypothesis " $s=h * s$ " in the Proposition 3.6.

Figure 3 displays zooms of an initial image (see Figure $3(\mathrm{a})$ ) which is cylindric along the direction $(1,3)$. Note that we display only the same extracted part of the zoomed images. Images $3(\mathrm{~b})$ and $3(\mathrm{c})$, which correspond to the duplication and the bicubic reconstructions, do present oscillating edges. In the same time, images 

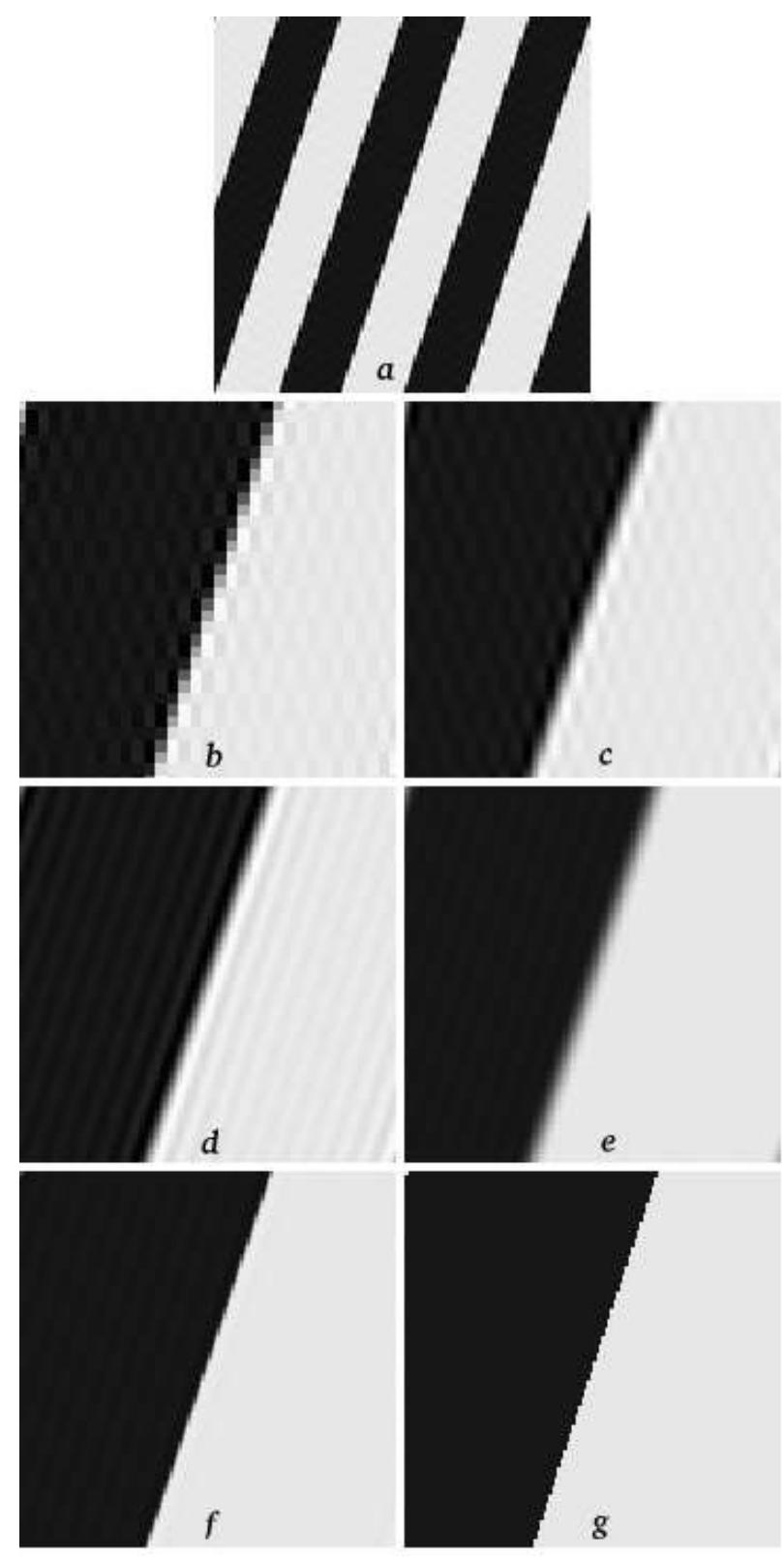

FIG. 3. (a) The initial image (cylindric along the direction $(1,3)$ ). Part of the images zoomed by a factor 4 by (b) the duplication interpolation; (c) the cubic convolution reconstruction; (d) the "zero-padding" interpolation; (e) the reconstruction using the prolate function; (f) the total variation based restoration. (g) Part of the reference image.

3(d) and 3(e), which correspond to the "zero-padding" and the linear reconstruction defined by the prolate function, do not. This illustrates Proposition 2.9, since the convolution kernels associated with the duplication and bicubic reconstructions do not have Fourier transforms supported on $]-\frac{1}{2}, \frac{1}{2}[2$, while the "sinc" and the prolate functions do. In the same way, image $3(\mathrm{f})$ is a part of the total variation based restoration associated with the "sinc function" $\left(s=s^{e}\right)$ and does not have oscillating 


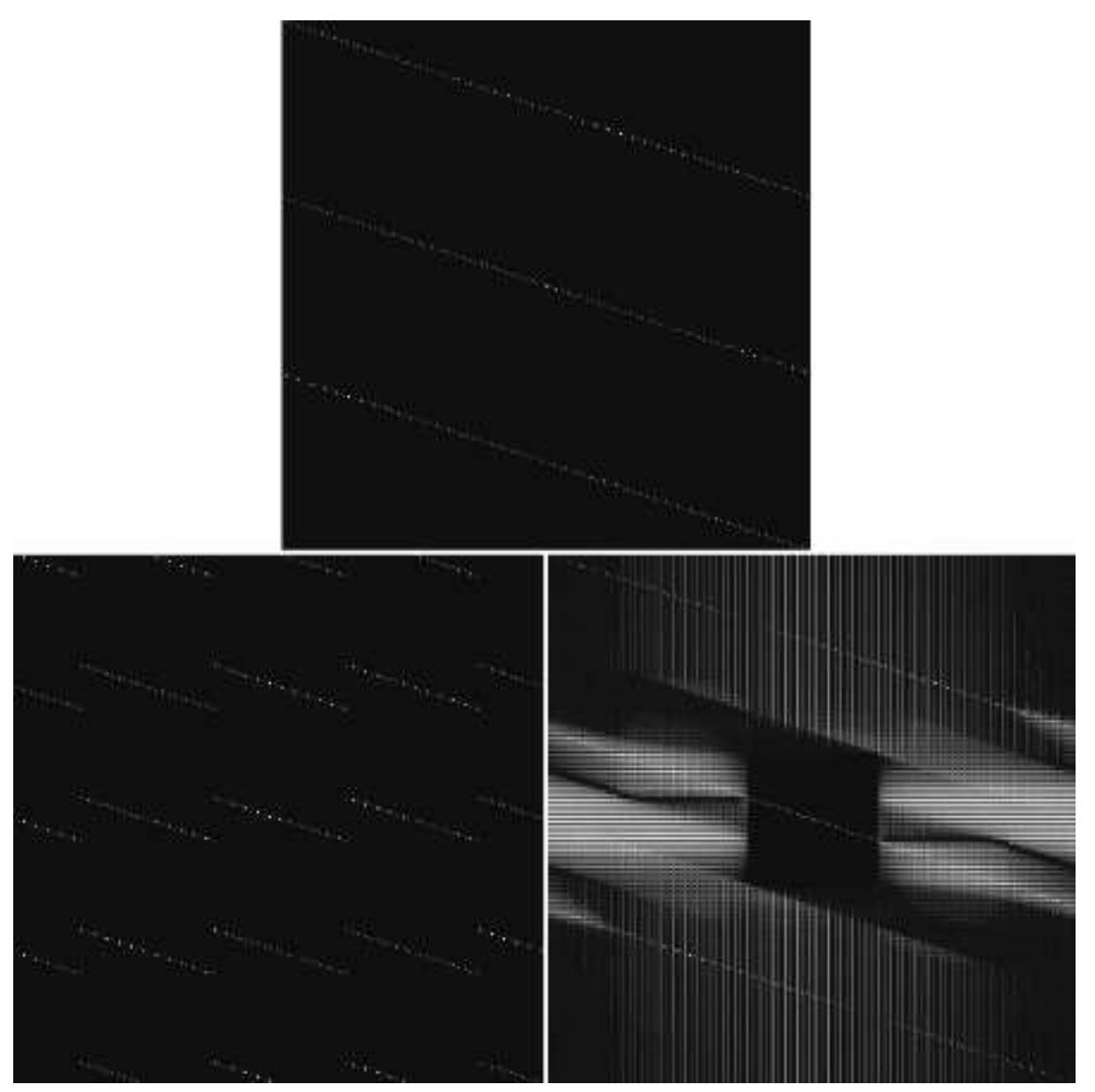

FIG. 4. Spectra of the images whose extracts are displayed on Figure 3. (a) the reference 3(g); (b) the duplication $3(\mathrm{~b})$; (c) the total variation based restoration $3(\mathrm{f})$.

edges. Moreover, image 3(d) oscillates more than the initial image $3(\mathrm{~g})$, while image 3 (e) does not (but it is very blurred). We can see that the total variation restoration (Figure 3(f)), owing to its ability to extrapolate frequencies, has a sharp result which oscillates less than the initial image $3(\mathrm{~g})$.

Figure 4 represents the Fourier series of some reconstruction of the image 3(a) (whose extracts are displayed on Figures $3(\mathrm{~b}), 3(\mathrm{f})$, and $3(\mathrm{~g})$ ). We can see the periodical structure of Figure 4(b) which prevents the preservation of the 1D nature of image 3(a). Note that the same observation could have been made on the spectrum of the bicubic reconstruction. On the other hand, looking at Figure 4(c), we can see the ability of the total variation based restoration to prolong the initial structure out of $]-\frac{1}{2}, \frac{1}{2}\left[^{2}\right.$. ( $]-\frac{1}{2}, \frac{1}{2}\left[^{2}\right.$ is represented by the black square in the center of the figure.) Moreover, we observe on this spectrum the property announced in Proposition 3.9. Indeed, we took the same value for $K$ and $K^{\prime}$, and we can see that the Fourier series of the result is essentially supported on $S_{1,3}$ (see Proposition 3.9).

We display on Figure 5 the same zooming methods as the ones of Figure 3, in the case of an image representing a triangle. We can make about this figure the same 


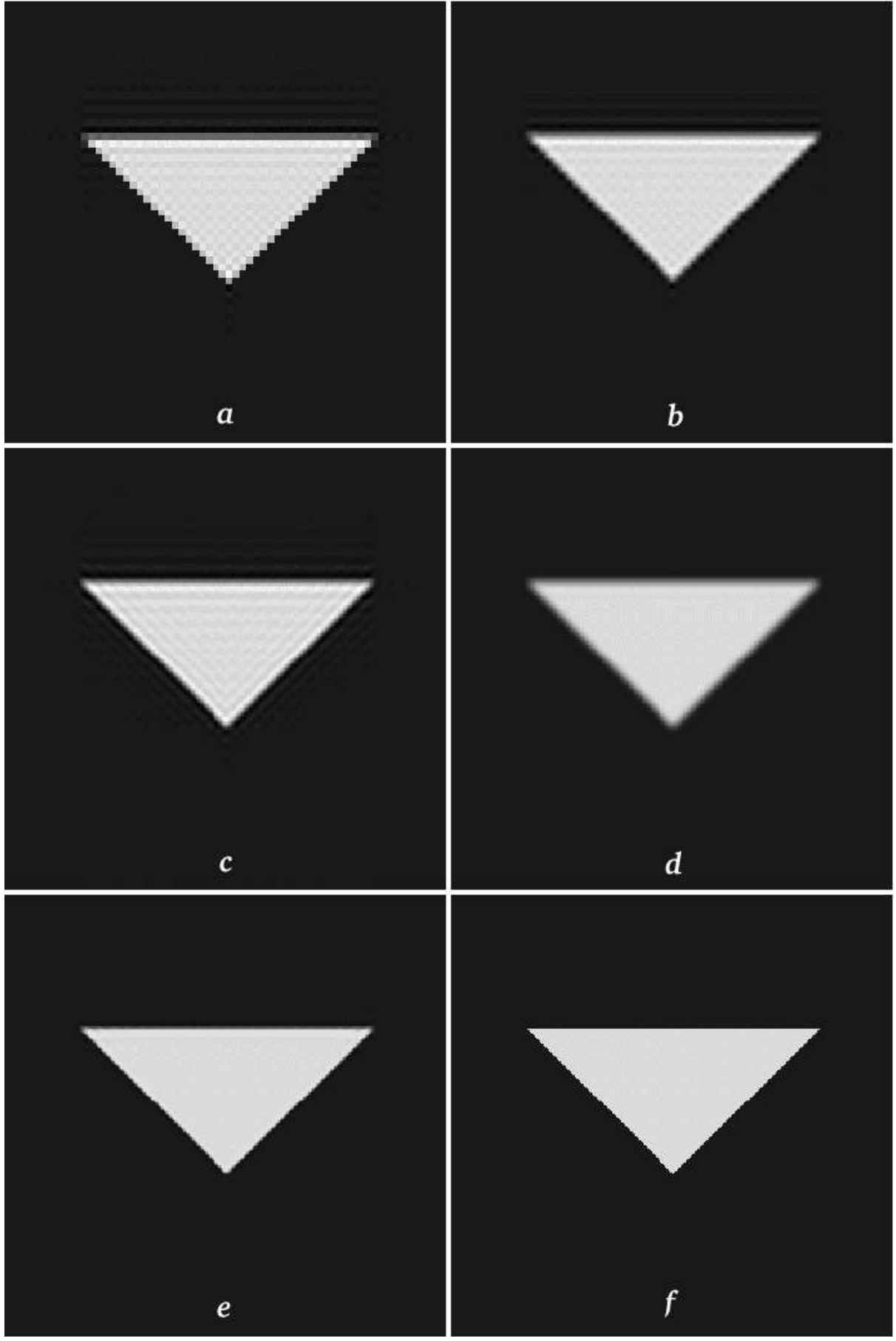

FIG. 5. Image zoomed by a factor 4 by (a) the duplication interpolation; (b) the cubic convolution reconstruction; (c) the "zero-padding" interpolation; (d) the reconstruction using the prolate function; (e) the total variation based restoration; (f) the reference image. 
comments as the one made about Figure 3. Moreover, we remark that the horizontal edge of the triangle is accurately restored by all the linear reconstructions. This is basically due to the fact that all the associated kernels satisfy the hypotheses of Proposition 2.7. Moreover, we can see a limit of our model on Figure 5(e). Indeed, we note some oscillations on the edges in the vicinity of the triangle corners. These are due to the need of the total variation to erode the corners in order to decrease. Figure 6 displays the spectrum of the images of Figure 5. Figures 6(a) and 6(b) illustrate Proposition 2.7. (The periodizations of the vertical white line are located at the same places as the vertical black ones; therefore they are canceled and the horizontal edge is correctly restored). Moreover, Figure 6(e) shows that the total variation restoration is the only one which tends to prolong the initial structures of the spectrum.

We display in Figure 7 the same zooming methods as the ones of Figure 3, in the case of an image representing an ellipse. The ellipse is clearly a limit case of the cylindric model, since the curvature of its edge is never null. This experiment shows that the results based on the modeling of edges by cylindric functions still apply to other kinds of edges, since the conclusions of the preceding experiments still hold. Moreover, looking at Figures $8(\mathrm{e})$ and $8(\mathrm{f})$, we see that the total variation based restoration permits one to extend the initial spectrum much better than the duplication or any other linear method that periodizes it.

In order to estimate the relevance of our analysis in the case of a real world image, we display in Figure 9 the same reconstruction/restoration methods in the case of an image provided by the Centre National d'Etudes Spatiales. A part of this image presents oscillations that are essentially cylindric and for which the comments made for Figure 3 are valid. Moreover, this oscillating zone is almost lost on Figures 9 (a) and 9(b). This artifact is due to the spectrum periodization and is similar to the artifacts usually caused by aliasing. (We added another oscillation in a wrong direction.) Furthermore, Figure 9(e) shows a shortcoming of the total variation based restoration, that is, that it tends to create homogeneous zones. We display in Figure 10 the spectra of the images of Figure 9. We observe here the frequency translation of most of the artifact seen on the spatial representation. In particular, the ability of the total variation based restoration to preserve cylindric images is translated in its ability to prolong the initial spectrum structure to the new spectral domain. However, we see in Figures 10(e) and 10(f) that, even if we extend the structures present in the original spectrum, the values, given a direction, are different.

We enumerate in the Table 3 the mean square error between the reconstruction and the reference images. We see that, among linear reconstruction, the zero-padding always yields the best statistics (which seems normal given the sampling process yielding the data). Moreover, the total variation based restoration yields better results than linear ones. These statistics are strongly improved in the cases of artificial images while this difference is smaller in the case of the natural image. This is classical, but we could expect to have better statistics for natural images presenting many sharp edges.

At last, Figure 11 shows evidence of the importance of the hypothesis " $s=h * s^{e}$ " in Proposition 3.6. It represents a small part of the zooms of level 8 of a natural image (a photograph of an eye; note that the contrast has been highly modified). The difference in all these total variation based restorations holds in the filter $s$ used to define $\mathcal{W}_{s, u}$ (up-down: the size of the frequencial support of $s$ decreases; see the right part of Figure 11). On Figure 11(a), the values are fixed for each of every 8 pixels, the other ones are free of any constraint $(\hat{s}=1)$; the "number of constraint points" increases until Figure 11(d), where the constraint corresponds to the case $s=s^{e}$ 

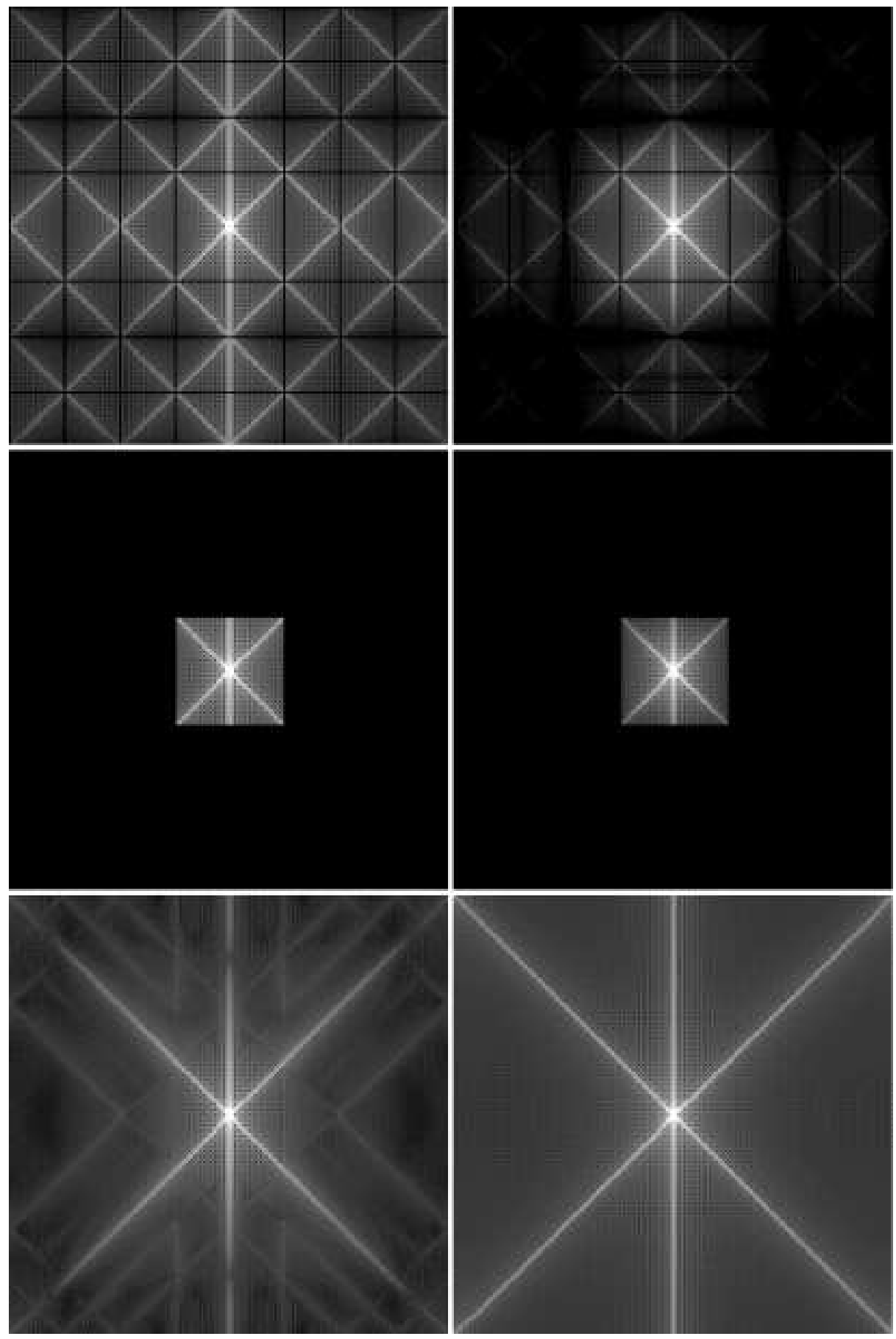

FIG. 6. Spectra of the images displayed on Figure 5. 

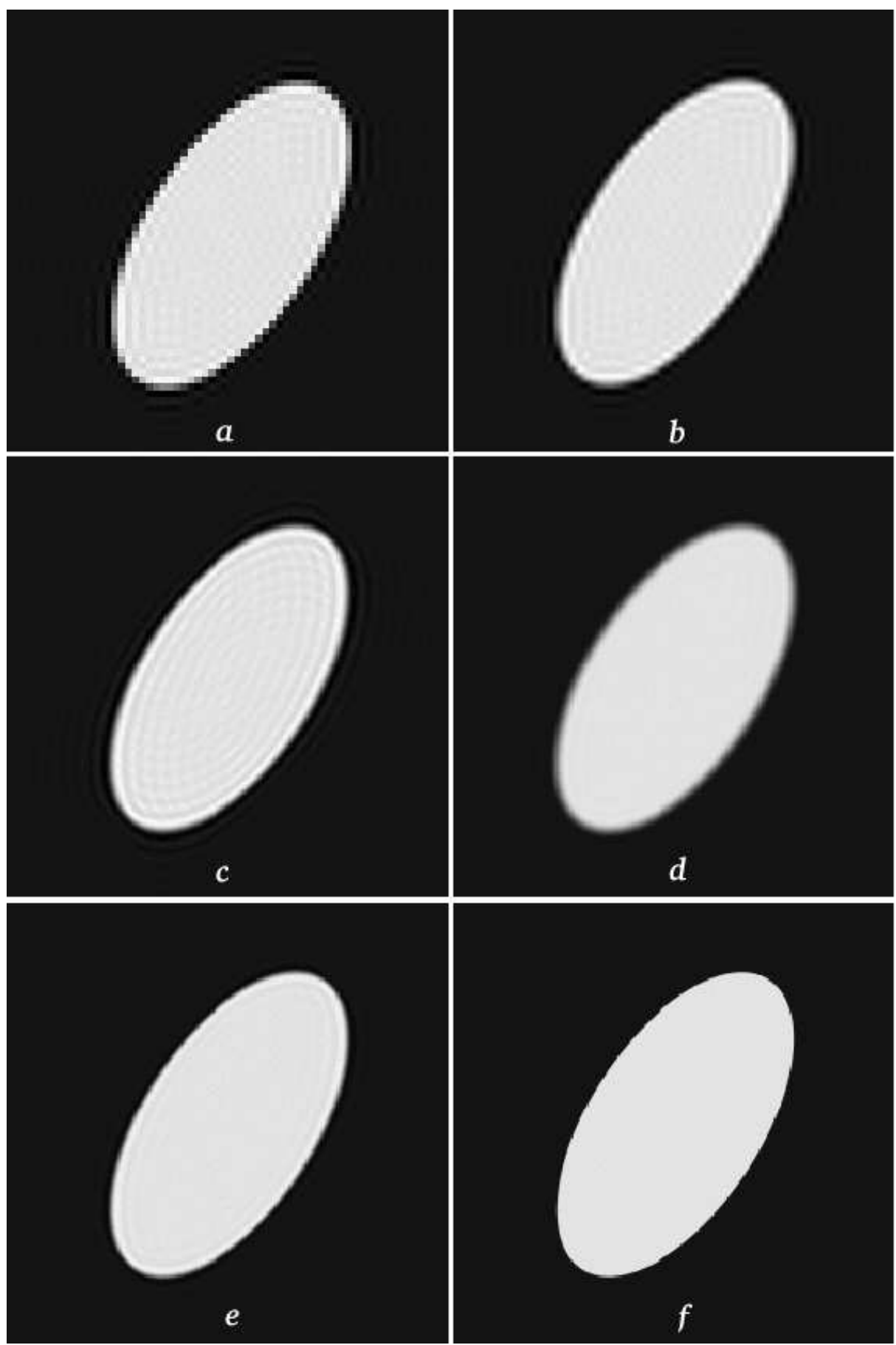

FIG. 7. Image zoomed by a factor 4 by (a) the duplication interpolation; (b) the cubic convolution reconstruction; (c) the "zero-padding" interpolation; (d) the reconstruction using the prolate function; (e) the total variation based restoration; (f) the reference image. 


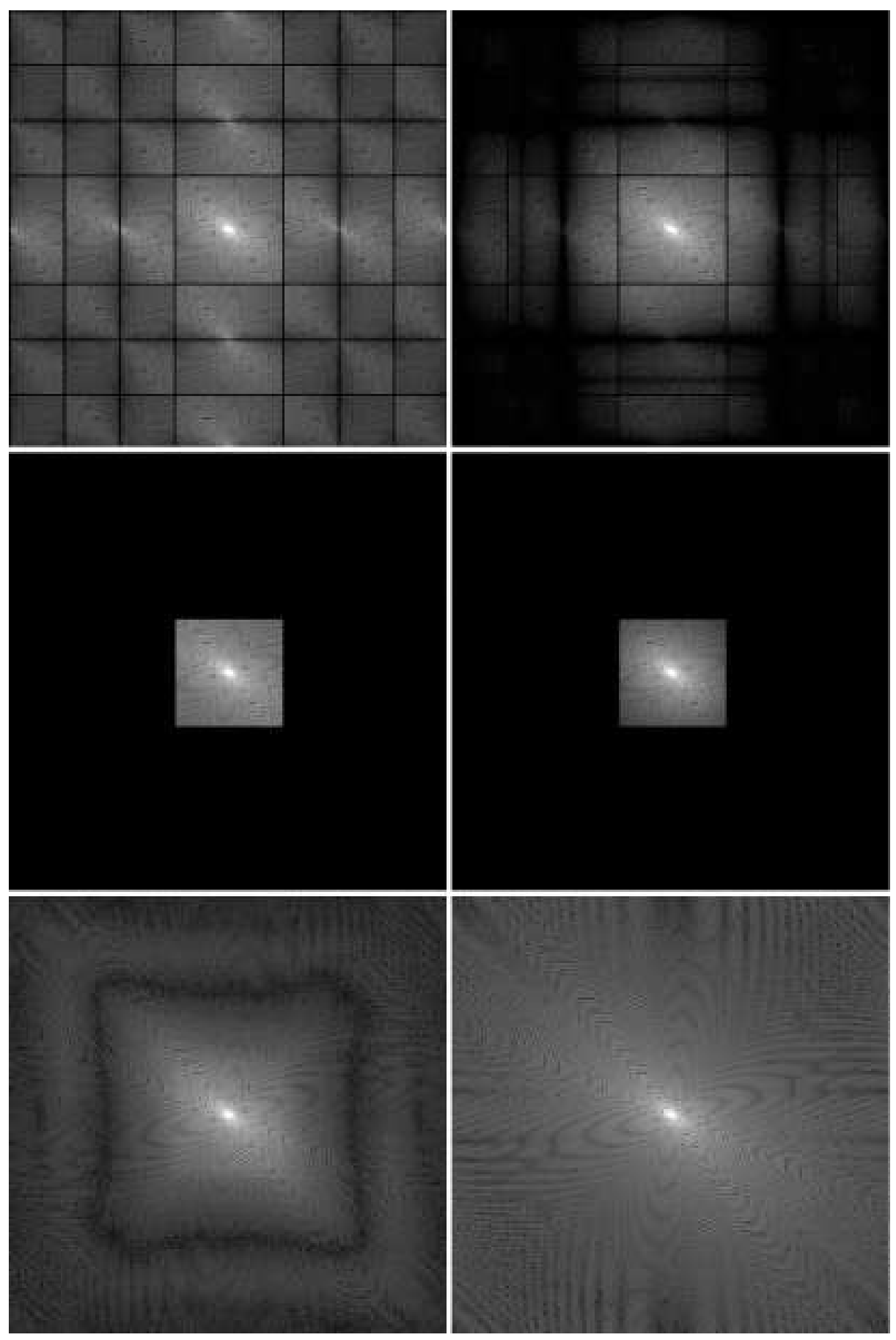

Fig. 8. Spectra of the images displayed on Figure 7. 

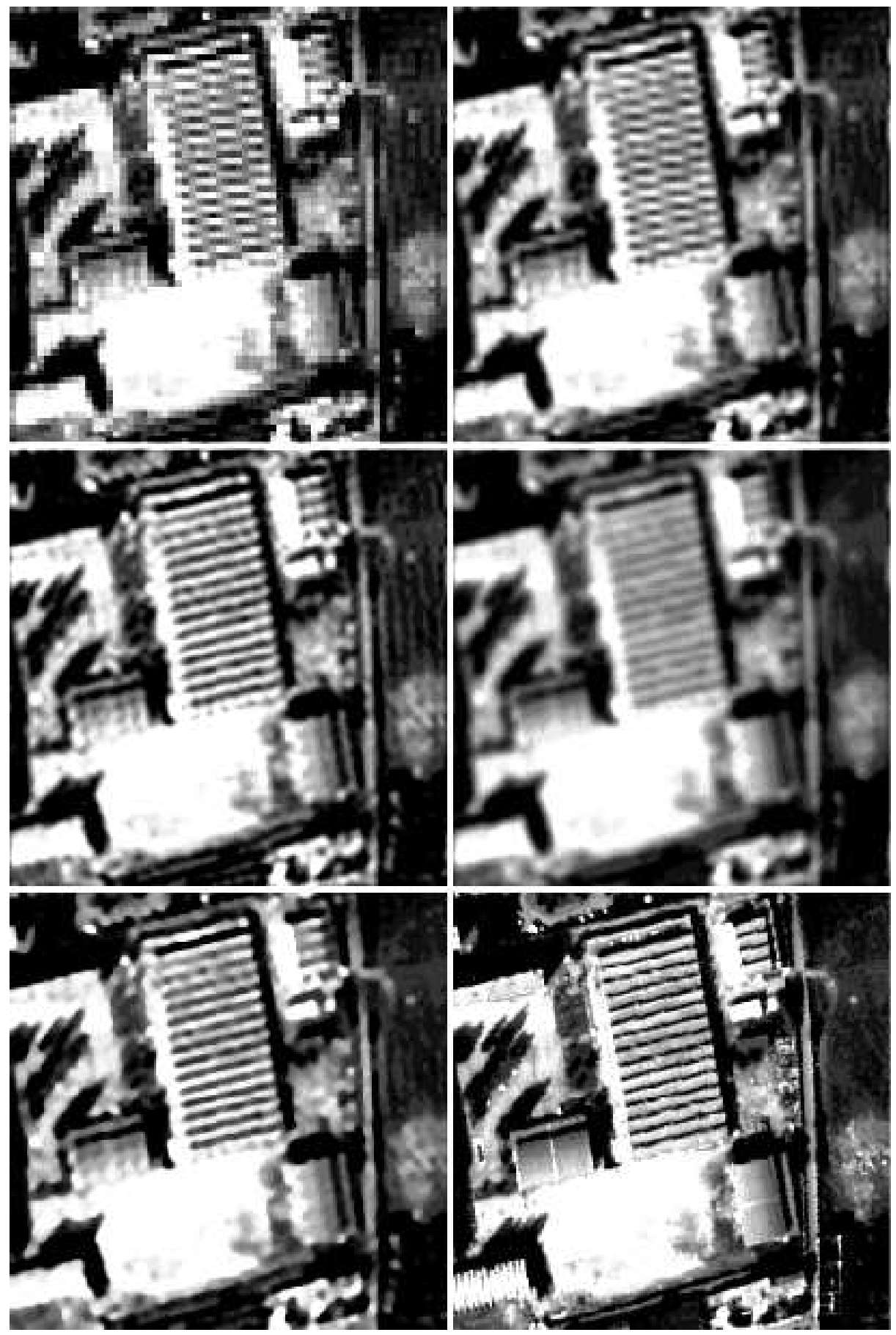

FIG. 9. Image zoomed by a factor 4 by (a) the duplication interpolation; (b) the cubic convolution reconstruction; (c) the "zero-padding" interpolation; (d) the reconstruction using the prolate function; (e) the total variation based restoration; (f) the reference image. 


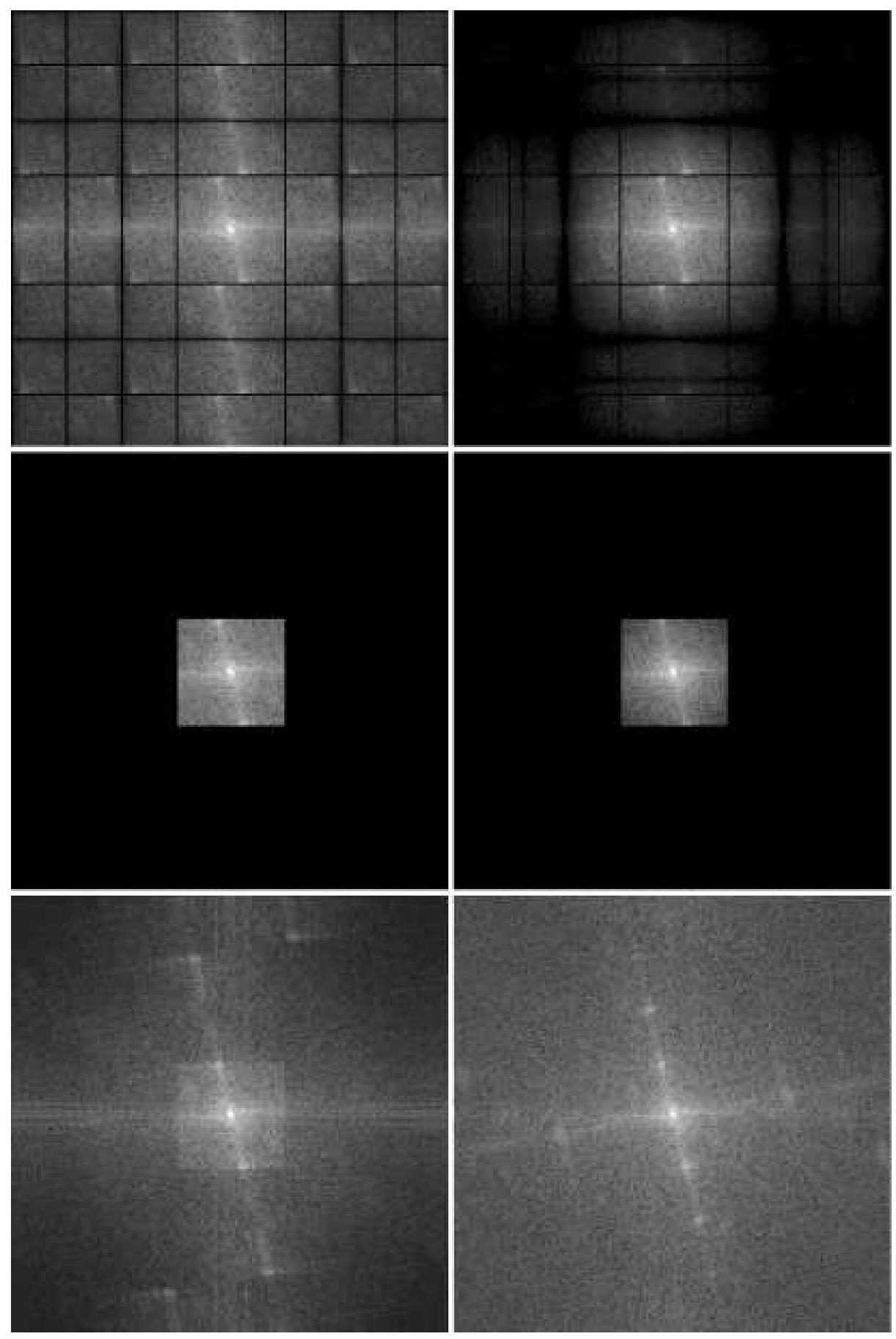

FIG. 10. Spectra of the images displayed on Figure 9. 

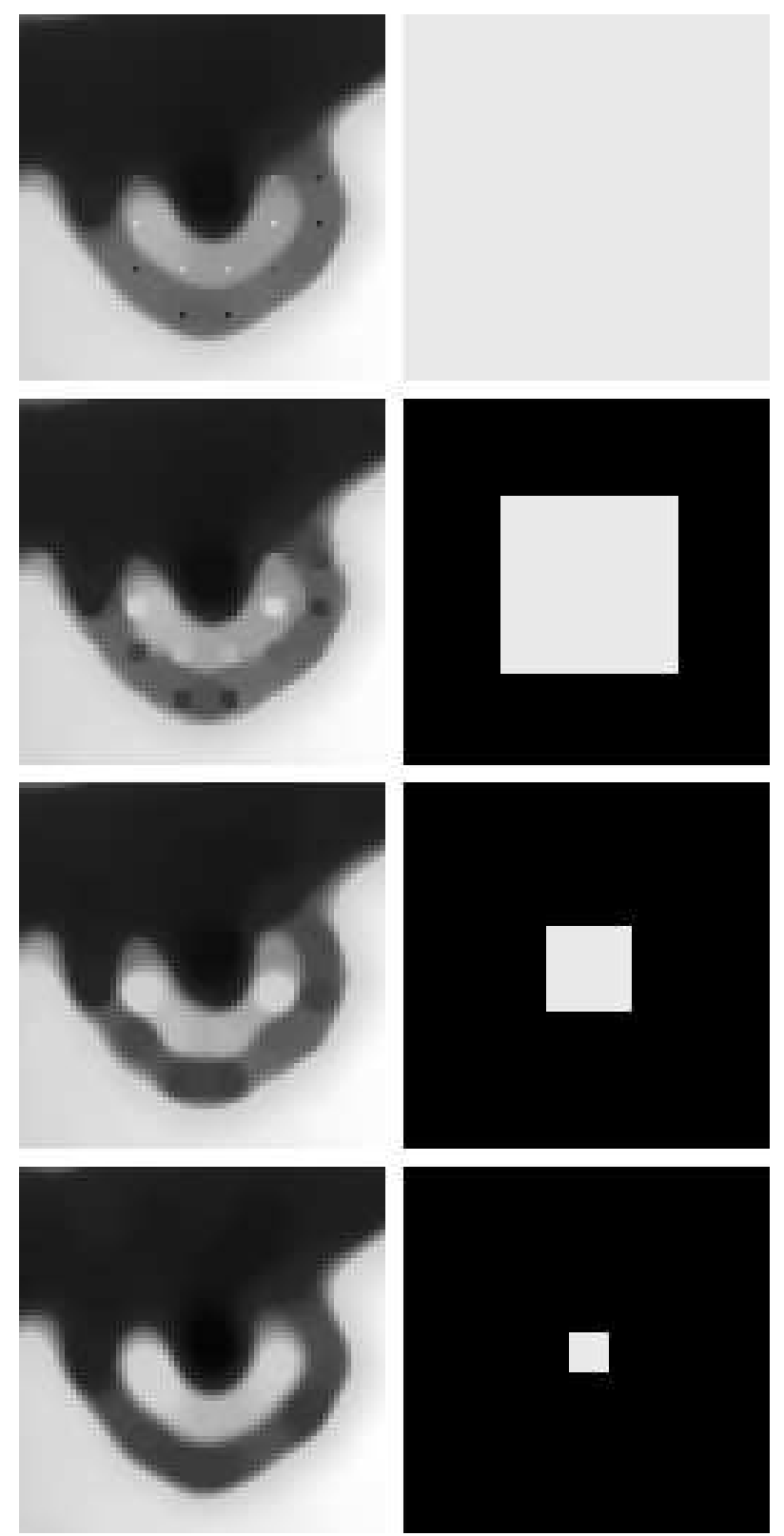

FIG. 11. Left: the images restored with the total variation restoration (zooms of level 8; the contrast has been modified). Right: the real part of the Fourier transform of the kernel $s$ used to restore the image on the left (i.e., this latter belongs to $\left.\mathcal{W}_{s, u}\right)$.

(see Proposition 3.6). We see in Figure 11 that the zones that suffer "significant" constraint are visible on Figures 11(a), 11(b), 11(c), but not in Figure 11(d). This is due to the fact that the points for which the constraint is too weak collapse in order to make the total variation decrease. 
TABLE 3

Mean square error between the reconstruction and the reference image.

\begin{tabular}{|c|c|c|c|c|c|c|}
\hline Image & Figure & $\begin{array}{c}\text { Duplication } \\
\text { interpolation }\end{array}$ & $\begin{array}{c}\text { Bicubic } \\
\text { rec. }\end{array}$ & $\begin{array}{c}\text { Zero- } \\
\text { padding }\end{array}$ & $\begin{array}{c}\text { Prolate } \\
\text { rec. }\end{array}$ & $\begin{array}{c}\text { Variational } \\
\text { restoration }\end{array}$ \\
\hline Cylindric & Fig. 3 & 928 & 378 & 347 & 465 & 224 \\
\hline Triangle & Fig. 5 & 522 & 165 & 133 & 202 & 56 \\
\hline Ellipse & Fig. 7 & 92.03 & 20.06 & 16.30 & 26.97 & 8.88 \\
\hline Natural & Fig. 9 & 289 & 124 & 102 & 148 & 99 \\
\hline
\end{tabular}

Acknowledgments. The authors would like to thank J.M. Morel, B. Rougé, and S. Durand for many fruitful discussions on that subject.

\section{REFERENCES}

[1] R. ACAR AND C. Vogel, Analysis of bounded variation methods for ill-posed problems, Inverse Problems, 10 (1994), pp. 1217-1229.

[2] A. Albiol and J. Serra, Morphological image enlargements, J. Visual Comm. and Image Representation, 8 (1997), pp. 367-383.

[3] J. Allebach and P. W. Wong, Edge-directed interpolation, in Proceedings of the International Conference on Image Processing, Vol. 3, IEEE Press, Los Alamitos, CA, 1996, pp. 707-710.

[4] E. Casas, K. Kunisch, And C. Pola, Regularization by functions of bounded variation and applications to image enhancement, Appl. Math. Optim., 40 (1999), pp. 229-257.

[5] V. Caselles, J. L. Lisani, J. M. Morel, and G. Sapiro, Shape preserving local histogram modification, IEEE Trans. Image Process., 8 (1999), pp. 220-230.

[6] A. Chambolle And P.-L. Lions, Image recovery via total variation minimization and related problems, Numer. Math., 76 (1997), pp. 167-188.

[7] T. F. Chan And P. Mulet, On the convergence of the lagged diffusivity fixed method in total variation image restoration, SIAM J. Numer. Anal., 36 (1999), pp. 354-367.

[8] S. Durand, F. Malgouyres, and B. Rougé, Image de-blurring, spectrum interpolation and application to satellite imaging, ESAIM Control Optim. Calc. Var., 5 (2000), pp. 445-475; also available at http://www.cmla.ens-cachan.fr/ malgouy.

[9] L. Evans and R. F. Gariepy, Measure Theory and Fine Properties of Functions, Stud. Adv. Math., CRC Press, Boca Raton, FL, 1992.

[10] F. Guichard And F. Malgouyres, Total variation based interpolation, in Proceedings of the Ninth European Signal Processing Conference, Vol. 3, Patran, Greece, 1998, pp. 1741-1744.

[11] K. Iто AND K. KUNISCH, An active set strategy based on the augmented Lagrangian formulation for image restoration, Math. Model. Numer. Anal., 33 (1999), pp. 1-21.

[12] N. B. KARAYIANNIS AND A. N. VenEtSNOPOULOS, Image interpolation based on variational principles, Signal Processing, 25 (1991), pp. 259-288.

[13] H. Landau and H. Pollak, Prolate spheroidal wave functions, Fourier analysis and uncertainty III: The dimension of the space of essentially time and bandlimited signals, Bell System Tech. J., 41 (1962), pp. 1295-1336.

[14] E. Maeland, On the comparison of interpolation methods, IEEE Trans. Medical Imaging, 7 (1988), pp. 213-217.

[15] F. Malgouyres, Increase in the Resolution of Digital Images: Variational Theory and Applications, Ph.D. thesis, Ecole Normale Superieure de Cachan, Cachan, France, 2000, available at http://www.cmla.ens-cachan.fr/ malgouy.

[16] S. Mallat, A Wavelet Tour of Signal Processing, Academic Press, Boston, 1998.

[17] S. Matej, S. S. Furuie, and G. Herman, The relevance of statistically significant differences between reconstruction algorithms, IEEE Trans. Image Process., 5 (1996), pp. 554-556.

[18] M. NASHED AND O. ScheRZER, Least square and bounded variation regularization with nondifferentiable functions, Numer. Funct. Anal. Optim., 19 (1998), pp. 873-901.

[19] J. P. OACkley and M. J. Cunningham, A function space model for digital image sampling and its application in image reconstruction, Comput. Vision Graphics Image Process., 49 (1990), pp. 171-197.

[20] S. K. PARK And R. A. Schrowengerdt, Image reconstruction by parametric cubic convolution, Comput. Vision Graphics Image Process., 23 (1983), pp. 258-272.

[21] L. Rudin, S. Osher, ANd E. FATEMI, Nonlinear total variation based noise removal algorithms, Phys. D, 60 (1992), pp. 259-268. 
[22] L. I. Rudin, Images, Numerical Analysis of Singularities and Shock Filters, Ph.D. thesis, California Institute of Technology, Pasadena, CA, 1987.

[23] W. Rudin, Real and Complex Analysis, 3rd ed., McGraw-Hill, New York, 1987.

[24] C. E. Shannon, Communication in the presence of noise, Proceedings of the IRE, 37 (1949), pp. $10-21$.

[25] S. Thurnhofer and S. K. Mitra, Edge-enhanced image zooming, Optical Engrg., 35 (1996), pp. $1862-1870$.

[26] M. Unser, A. Aldroubi, And M. Eden, Enlargement or reduction of digital image with minimum loss of information, IEEE Trans. Image Process., 4 (1995), pp. 247-258.

[27] S. J. Wernecke and L. D'AdDario, Maximum entropy image reconstruction, IEEE Trans. Comput., c-26 (1977), pp. 351-364.

[28] L. Yaroslavsky, Efficient algorithm for discrete sinc interpolation, Appl. Optics, 36 (1997), pp. 460-463.

[29] K. YosidA, Functional Analysis, Springer-Verlag, Berlin, 1965.

[30] A. Zygmund, Trigonometric Series, Cambridge University Press, Cambridge, UK, 1959. 